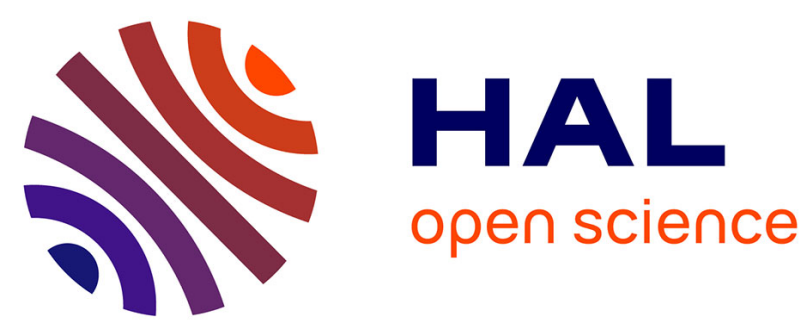

\title{
Opportunistic autoimmunity secondary to cancer immunotherapy (OASI): An emerging challenge
}

Marie Kostine, L Chiche, E Lazaro, P. Halfon, C Charpin, D Arniaud, F Retomaz, Patrick Blanco, Noemie Jourde-Chiche, C. Richez, et al.

\section{- To cite this version:}

Marie Kostine, L Chiche, E Lazaro, P. Halfon, C Charpin, et al.. Opportunistic autoimmunity secondary to cancer immunotherapy (OASI): An emerging challenge. La Revue de Médecine Interne, 2017, 38 (8), pp.513 - 525. 10.1016/j.revmed.2017.01.004 . hal-01791069

\section{HAL Id: hal-01791069 \\ https://hal-amu.archives-ouvertes.fr/hal-01791069}

Submitted on 28 Mar 2019

HAL is a multi-disciplinary open access archive for the deposit and dissemination of scientific research documents, whether they are published or not. The documents may come from teaching and research institutions in France or abroad, or from public or private research centers.
L'archive ouverte pluridisciplinaire HAL, est destinée au dépôt et à la diffusion de documents scientifiques de niveau recherche, publiés ou non, émanant des établissements d'enseignement et de recherche français ou étrangers, des laboratoires publics ou privés. 


\title{
Opportunistic autoimmunity secondary to cancer immunotherapy (OASI): An emerging challenge
}

\author{
M. Kostine ${ }^{\mathrm{a}}$, L. Chiche ${ }^{\mathrm{b}, *}$, E. Lazaro $^{\mathrm{c}}$, P. Halfon ${ }^{\mathrm{b}}$, , C. Charpin $^{\mathrm{b}, \mathrm{d}}$, D. Arniaud ${ }^{\mathrm{d}}$, F. Retornaz $^{\mathrm{b}}$, \\ P. Blanco ${ }^{e}$, N. Jourde-Chiche ${ }^{f}$, C. Richez ${ }^{\mathrm{a}}$, C. Stavris ${ }^{\mathrm{b}}$ \\ a Département de rhumatologie, hôpital Pellegrin, place Amélie-Raba-Léon, Bordeaux, France \\ b Service de médecine interne, département de médecine interne et infectiologie, hôpital européen de Marseille, 6, rue Désirée-Clary, Marseille, 13003 France \\ ${ }^{\mathrm{c}}$ Département de médecine interne, hôpital du Haut-Lévêque, Pessac, France \\ d Département de rhumatologie, hôpital Saint-Joseph, Marseille, France \\ e Département d'immunologie, hôpital Pellegrin, Bordeaux, France \\ ${ }^{\mathrm{f}}$ Département de néphrologie, hôpital de la conception, AP-HM, Marseille, France
}

\begin{abstract}
A B S T R A C T
With "checkpoint inhibitors" targeting PD1/PD-1-ligands or CTLA-4/CD28 pathways, immunotherapy has profoundly modified therapeutic strategies in oncology. First approved in refractory metastatic neoplasms (melanoma and lung adenocarcinoma), it is now being tested broadly in other cancers and/or as adjuvant treatment. For a significant proportion of patients, immunotherapy is responsible for "immunological" events, identified as Immune-Related Adverse Events (irAEs). Owing to the increasing number of prescriptions, identification and management of specific immunological side effects is crucial and requires close collaboration between oncologists and internists and/or other organ specialists. Within irAEs, we propose to individualize the induced autoimmunity by the term "Opportunistic Autoimmunity Secondary to Cancer Immunotherapy" (OASI). The aims of this article are (1) to present the different available checkpoint inhibitors and the OASIs reported with these treatments and (2) to propose practical recommendations for diagnosis, pre-therapeutic assessment and management of OASIs. The need for predictive biomarkers of OASIs occurrence will also be discussed.
\end{abstract}

Keywords:

Autoimmunity

Immunotherapy

Cancer

Opportunistic

Checkpoint inhibitors

\section{Opportunistic autoimmunity secondary to cancer immunotherapy}

\subsection{Background}

Recently, immunotherapy has completely modified the management of cancer patients. Of note, sustained remissions have been obtained in patients with cancers refractory to conventional approaches, such as metastatic melanoma [1-4]. After an initial use restricted to melanoma and lung adenocarcinoma [2-20], immunotherapy is now being tested in patients with many other types of cancers, as well as in first-line therapy for patients naive to chemotherapy. While the concept of immunotherapy in cancer is far from new, the monoclonal antibodies "checkpoint inhibitors" clearly marked a turning point in the success of this approach. The molecules currently available target CTLA-4 and PD-1

\footnotetext{
* Corresponding author.

E-mail address: 1.chiche@hopital-europeen.fr (L. Chiche).
}

receptors or their ligands, but several other immunomodulatory agents are under development $[21,22]$ (Fig. 1). So far, the results available (Table 1) indicate a broadening of indications to come; therefore, it is crucial to become familiar with these new molecules and with the management of their specific side effects.

Indeed, cancer immunotherapy is responsible for autoimmune manifestations in a significant proportion of patients (70 to $90 \%$ of patients receiving anti-PD- 1 and anti-CTLA-4 respectively), potentially severe ( $\leq 10 \%$ and $20 \%$ respectively). Diagnosis and management of these autoimmune events require a close collaboration between oncologists and internists and/or other organ specialists. In addition to their expertise for diagnosis, these specialists have experience using biologics such anti-TNF to manage induced/paradoxical autoimmunity and monitoring patients during and after immunotherapy to detect potential short- and long-term side effects. In the context of cancer immunotherapy, notably checkpoint inhibitors, T-cell activation is induced by releasing the PD-1/PD-1 ligands or CTLA-4/CD28 co-inhibitory signals, leading to a cytotoxic attack of tumor cells. However, this T-cell activation may also promote the emergence of autoimmunity 
Table 1

OASIs reported in anti-PD1 trials.

\begin{tabular}{|c|c|c|c|c|}
\hline Studies & Phase $n$ & Molecule/Dosage/ \pm Association & Cancer/Stage & Oncologic response \\
\hline Hamanishi et al., 2015 [5] & $\begin{array}{l}\text { II } \\
n=20\end{array}$ & Nivolumab 1 or $3 \mathrm{mg} / \mathrm{kg} / 2 \mathrm{~W}$ & Ovarian platines $\mathrm{R}$ & $\begin{array}{l}\text { CR } 2(10 \%) \\
\text { PR } 1(5 \%)\end{array}$ \\
\hline Patnaik et al., 2015 [6] & $\begin{array}{l}\mathrm{I} \\
n=30\end{array}$ & $\begin{array}{l}\text { Pembrolizumab } \\
\text { Escalade dose }\end{array}$ & Solid (MM, NSCLC...) & $\begin{array}{l}\text { CR } 2(7 \%) \\
\text { PR } 3(10 \%)\end{array}$ \\
\hline Rizvi et al., 2015 [7] & $\begin{array}{l}\text { II } \\
n=117\end{array}$ & $\begin{array}{l}\text { Nivolumab } \\
3 \mathrm{mg} / \mathrm{kg} / 2 \mathrm{~W}\end{array}$ & $\begin{array}{l}\text { NSCLC } \\
\mathrm{M}+\text { multi-R }\end{array}$ & $\begin{array}{l}\text { CR 0 (0\%) } \\
\text { PR } 17(15 \%)\end{array}$ \\
\hline Hamid et al., 2013 [4] & $\begin{array}{l}\mathrm{I} \\
n=135\end{array}$ & $\begin{array}{l}\text { Pembrolizumab } \\
10 \mathrm{mg} / \mathrm{kg} / 2 \text { or } 3 \mathrm{~W} \\
\text { Or } 2 \mathrm{mg} / \mathrm{kg} / 3 \mathrm{~W} \\
\pm \text { Ipilimumab }\end{array}$ & $\begin{array}{l}\mathrm{MM} \\
\mathrm{M}+\text { or unresectable }\end{array}$ & GR $44(38 \%)$ \\
\hline Garon et al., 2015 [8] & $\begin{array}{l}\mathrm{I} \\
n=495\end{array}$ & $\begin{array}{l}\text { Pemprolizumab } \\
2 \mathrm{mg} \text { or } 10 \mathrm{mg} / \mathrm{kg} / 3 \mathrm{~W} \\
\text { Or } 10 \mathrm{mg} / \mathrm{kg} / 2 \mathrm{~W}\end{array}$ & $\begin{array}{l}\text { NSCLC } \\
\text { Advanced or } \mathrm{M}^{+}\end{array}$ & ORR $19.4 \%$ \\
\hline Larkin et al., 2015 [3] & $\begin{array}{l}\mathrm{III} \\
n=945\end{array}$ & $\begin{array}{l}\text { Nivolumab } \\
\text { Variable dosage } \pm \text { Ipilimumab } \\
\mathrm{N} n=316 \\
\mathrm{~N}+\mathrm{I} n=314\end{array}$ & $\begin{array}{l}\text { MM } \\
\text { III or IV }\end{array}$ & $\begin{array}{l}\text { CR } 4(1 \%) \\
\text { PR } 255(40 \%)\end{array}$ \\
\hline Brahmer et al., 2015 [9] & $\begin{array}{l}\mathrm{III} \\
n=272\end{array}$ & $\begin{array}{l}\text { Nivolumab } \\
3 \mathrm{mg} / \mathrm{kg} / 2 \mathrm{~W} \\
\mathrm{~N} n=135\end{array}$ & $\begin{array}{l}\mathrm{NSCLC} \\
\mathrm{M}^{+}\end{array}$ & $\begin{array}{l}\text { CR 1 (1\%) } \\
\text { PR 26 (19\%) }\end{array}$ \\
\hline Gettinger et al., 2015 [10] & $n=129$ & $\begin{array}{l}\text { Nivolumab } \\
1 \text { or } 3 \text { or } 10 \mathrm{mg} / \mathrm{kg} / 2 \mathrm{~W} \\
\text { Escalade dose }\end{array}$ & $\begin{array}{l}\mathrm{NSCLC} \\
\mathrm{M}^{+}\end{array}$ & ORR $22(17 \%)$ \\
\hline McDermott et al., 2015 [11] & $\begin{array}{l}\mathrm{I} \\
n=34\end{array}$ & $\begin{array}{l}\text { Nivolumab } \\
1 \text { or } 10 \mathrm{mg} / \mathrm{kg} / 2 \mathrm{~W}\end{array}$ & $\begin{array}{l}\text { Renal } \\
\text { advanced }\end{array}$ & ORR $10(29 \%)$ \\
\hline Robert et al., 2015 [12] & $\begin{array}{l}\mathrm{III} \\
n=210\end{array}$ & $\begin{array}{l}\text { Nivolumab } \\
3 \mathrm{~g} / \mathrm{kg} / 2 \mathrm{~W} \\
(n=210)\end{array}$ & $\begin{array}{l}\mathrm{MM} \\
\mathrm{M}^{+}\end{array}$ & $\begin{array}{l}\text { CR } 16(8 \%) \\
\text { PR } 68(32 \%)\end{array}$ \\
\hline Topalian et al., 2014 [13] & $\begin{array}{l}\mathrm{I} \\
n=107\end{array}$ & $\begin{array}{l}\text { Nivolumab } \\
1 \text { or } 3 \text { or } 10 \mathrm{mg} / \mathrm{kg} / 2 \mathrm{~W} \\
\text { Escalade dose }\end{array}$ & $\begin{array}{l}\text { MM } \\
\text { advanced }\end{array}$ & ORR 33 (31\%) \\
\hline Ansell et al., 2015 [14] & $\begin{array}{l}\mathrm{I} \\
n=23\end{array}$ & $\begin{array}{l}\text { Nivolumab } \\
3 \mathrm{mg} / \mathrm{kg} / 2 \mathrm{~W} \\
\text { Escalade dose }\end{array}$ & $\begin{array}{l}\text { Hodgkin } \\
\text { Relapsing or } \mathrm{R}\end{array}$ & $\begin{array}{l}\text { CR } 4(17 \%) \\
\text { PR } 16(70 \%)\end{array}$ \\
\hline Motzer et al., 2015 [15] & $\begin{array}{l}\text { II } \\
n=168\end{array}$ & $\begin{array}{l}\text { Nivolumab } \\
0,3 \text { or } 2 \text { or } 10 \mathrm{mg} / \mathrm{kg} / 3 \mathrm{~W}\end{array}$ & $\begin{array}{l}\text { Renal } \\
\mathrm{M}^{+}\end{array}$ & $\begin{array}{l}\text { CR 2 (1\%) } \\
\text { PR } 33(20 \%)\end{array}$ \\
\hline Le et al., 2015 [16] & $\begin{array}{l}\text { II } \\
n=41\end{array}$ & $\begin{array}{l}\text { Pembrolizumab } \\
10 \mathrm{mg} / \mathrm{kg} / 2 \mathrm{~W}\end{array}$ & $\begin{array}{l}\text { Solid (colorectal, endometrial, } \\
\text { small bowel, gastric) }\end{array}$ & $\begin{array}{l}\text { CR } 1(3 \%) \\
\text { PR } 8(23 \%)\end{array}$ \\
\hline Postow et al., 2015 [17] & $\begin{array}{l}\mathrm{I} \\
n=142\end{array}$ & $\begin{array}{l}\text { Nivolumab } \\
\text { Dose escalade } \\
\pm \text { Ipilimumab }(n=95)\end{array}$ & $\begin{array}{l}\mathrm{MM} \\
\mathrm{M}^{+}\end{array}$ & $\begin{array}{l}n+\mathrm{I} \\
\text { CR } 21(22 \%) \\
\text { PR } 37(39 \%)\end{array}$ \\
\hline Robert et al., 2014 [2] & $\begin{array}{l}\mathrm{I} \\
n=173\end{array}$ & $\begin{array}{l}\text { Pembrolizumab } \\
2 \text { or } 10 \mathrm{mg} / \mathrm{kg} / 3 \mathrm{~W} \\
+ \text { Ipilimumab }\end{array}$ & $\begin{array}{l}\text { MM } \\
\text { Advanced }\end{array}$ & $\begin{array}{l}\text { CR } 2(1 \%) \\
\text { PR } 39(25 \%)\end{array}$ \\
\hline Robert et al., 2015 [18] & $\begin{array}{l}\mathrm{III} \\
n=834\end{array}$ & $\begin{array}{l}\text { Pembrolizumab } \\
10 \mathrm{mg} / \mathrm{kg} / 2 \text { or } 3 \mathrm{~W} \\
\mathrm{P} 2 \mathrm{~W} n=279 \\
\text { P3 } n=277\end{array}$ & $\begin{array}{l}\text { MM } \\
\text { Advanced III or IV }\end{array}$ & $\begin{array}{l}\text { GR/CR } \\
\text { P2W 34\%/5\% } \\
\text { P3W 33\%/6\% }\end{array}$ \\
\hline Wolchok et al., 2013 [19] & $\begin{array}{l}\mathrm{I} \\
n=86\end{array}$ & $\begin{array}{l}\text { Nivolumab } \\
\text { Dose Escalade } \\
+ \text { Ipilimumab } \\
\mathrm{N}+\mathrm{I} n=53 \\
\text { I then } \mathrm{N} n=33\end{array}$ & $\begin{array}{l}\text { MM } \\
\text { Advanced III or IV }\end{array}$ & $\begin{array}{l}\text { CR } 5(9 \%) \\
\text { PR } 16(30 \%)\end{array}$ \\
\hline Borghae et al., 2015 [20] & $\begin{array}{l}\mathrm{III} \\
n=292\end{array}$ & $\begin{array}{l}\text { Nivolumab } \\
3 \mathrm{mg} / \mathrm{kg} / 2 \mathrm{~W} n=292\end{array}$ & $\begin{array}{l}\text { NSCLC } \\
\text { IIIB or IV or relapsing }\end{array}$ & $\begin{array}{l}\text { CR 4 (1\%) } \\
\text { PR } 52(18 \%)\end{array}$ \\
\hline
\end{tabular}




\begin{tabular}{|c|c|c|c|c|c|c|c|c|c|c|}
\hline \multirow[t]{3}{*}{ Studies } & \multicolumn{10}{|l|}{ OASI } \\
\hline & \multirow{2}{*}{$\begin{array}{l}\text { Median age } \\
\text { Gender } \\
\text { Male (\%) } \\
\end{array}$} & \multicolumn{6}{|c|}{ Type and incidence $n(\%)^{\mathrm{a}}$} & \multicolumn{3}{|l|}{ Severity } \\
\hline & & Digestive & Hepatic & Endocrine & PNP & Cutaneous & Others & Grade 3-4 & $\begin{array}{l}\text { Death related } \\
\text { to OASI }\end{array}$ & $\begin{array}{l}\text { IT } \\
\text { discontinuation }\end{array}$ \\
\hline $\begin{array}{l}\text { Hamanishi et al., } \\
2015[5]\end{array}$ & $\begin{array}{l}60 \\
0 \%\end{array}$ & Frequent & $\begin{array}{l}\text { ASAT } 8(40 \%) \\
\text { ALAT } 5(25 \%)\end{array}$ & $\begin{array}{l}\text { HypoT } 8(40 \%) \\
\text { hyperT } 1(5 \%)\end{array}$ & $\mathrm{NA}$ & Rash $5(5 \%)$ & $\begin{array}{l}\text { Anemia } 4(20 \%) \text { arthralgia } \\
5(25 \%)\end{array}$ & $\begin{array}{l}\text { ALAT } 1 \text { (5\%) anemia } 3(15 \%) \\
\text { Rash } 1(5 \%)\end{array}$ & 0 & $\begin{array}{l}\text { Thyroiditis } 2 \\
(11 \%)\end{array}$ \\
\hline $\begin{array}{l}\text { Patnaik et al., } \\
2015[6]\end{array}$ & $\begin{array}{l}66.5 \\
77 \%\end{array}$ & Diarrhea $2(7 \%)$ & $0(0 \%)$ & HypoT 2 (7\%) & $1(3 \%)$ & $\begin{array}{l}\text { Erythema } 1(3 \%) \\
\text { Pruritus } 5(17 \%) \\
\text { Vitiligo } 1(3 \%)\end{array}$ & Myalgia $1(3 \%)$ & 0 & 0 & $1(3 \%)$ \\
\hline $\begin{array}{l}\text { Rizvi et al., } 2015 \\
\text { [7] }\end{array}$ & $\begin{array}{l}65 \\
73 \%\end{array}$ & $\begin{array}{l}\text { Diarrhea } 12 \\
(10 \%)\end{array}$ & Frequent & NA & $6(5 \%)$ & $\begin{array}{l}\text { Rash } 13(11 \%) \\
\text { Pruritus } 7(6 \%)\end{array}$ & $\begin{array}{l}\text { Anemia } 7(6 \%) \\
\text { Myalgia } 6(5 \%) \\
\text { Nephritis } 4(3 \%) \\
\text { Xerostomia } 7(6 \%)\end{array}$ & $\begin{array}{l}\text { Anemia } 1(\leq 1 \%) \\
\text { Diarrhea 3 }(3 \%) \\
\text { Rash 1 }(\leq 1 \%) ; \text { Prurit } 1(\leq 1 \%) \\
\text { Myalgia 1 }(\leq 1 \%) \\
\text { PNP 4 (3\%) }\end{array}$ & 1 (PNP) & $\begin{array}{l}\text { PNP } 5(4 \%) \\
\text { Adrenal1 }(\leq 1 \%) \\
\text { Diarrhea } 1 \\
(\leq 1 \%) \\
\text { NRP } 2(2 \%) \\
\text { Rash } 1(\leq 1 \%)\end{array}$ \\
\hline $\begin{array}{l}\text { Hamid et al., } \\
2013[4]\end{array}$ & $\begin{array}{l}60 \\
59 \%\end{array}$ & $\begin{array}{l}\text { Diarrhea } 27 \\
(20 \%)\end{array}$ & $\begin{array}{l}\text { ASAT } 13(10 \%) \\
\text { ALAT } 11(8 \%)\end{array}$ & $\begin{array}{l}\text { HypoT } 1(\leq 1 \%) \\
\text { HyperT } 1(\leq 1 \%) \\
\text { Adrenal } 1(\leq 1 \%)\end{array}$ & $6(4 \%)$ & $\begin{array}{l}\text { Rash } 28(21 \%) \\
\text { Pruritus } 28(21 \%) \\
\text { Vitiligo } 12(9 \%)\end{array}$ & Nephritis $3(2 \%)$ & $\begin{array}{l}\text { hypoT } 1(\leq 1 \%) \\
\text { hyperT } 1(\leq 1 \%) \\
\text { diarrhea } 1(\leq 1 \%) \\
\text { ASAT } 2(\leq 1 \%) \\
\text { Nephritis } 2(\leq 1 \%) \\
\text { Rash } 3(2 \%) \text { Pruritus } 1(\leq 1 \%)\end{array}$ & 1 (PNP) & $\mathrm{NA}$ \\
\hline $\begin{array}{l}\text { Garon et al., } 2015 \\
\text { [8] }\end{array}$ & NA & $\begin{array}{l}\text { Diarrhea } 40 \\
(8 \%)\end{array}$ & $\begin{array}{l}\text { ASAT } 15(3 \%) \\
\text { ALAT } 11(2 \%)\end{array}$ & НypoT 34 (7\%) & $18(4 \%)$ & $\begin{array}{l}\text { Rash } 48(10 \%) \\
\text { Pruritus } 53(11 \%)\end{array}$ & $\begin{array}{l}\text { Anemia } 21(4 \%) \\
\text { Arthralgia } 45(9 \%) \\
\text { Myalgia } 13(3 \%)\end{array}$ & $\begin{array}{l}\text { Rash } 1(\leq 1 \%) \\
\text { Arthralgia } 2(\leq 1 \%) \\
\text { Diarrhea } 3(\leq 1 \%) \\
\text { hypoT } 1(\leq 1 \%) \\
\text { PNP } 9(2 \%) \\
\text { ASAT } 3(\leq 1 \%) \text {; ALAT } 2(\leq 1 \%)\end{array}$ & 1 (PNP) & Yes \\
\hline $\begin{array}{l}\text { Larkin et al., } 2015 \\
\text { [3] }\end{array}$ & $\begin{array}{l}59 \\
65 \%\end{array}$ & $\begin{array}{l}\text { Diarrhea } 198 \\
(32 \%) \\
\text { Colitis } 41(7 \%)\end{array}$ & $\begin{array}{l}\text { ASAT } 60(10 \%) \\
\text { ALAT } 67(11 \%)\end{array}$ & HypoT 74 (12\%) & Frequent & $\begin{array}{l}\text { Pruritus } 163(26 \%) \\
\text { Rash } 207(33 \%)\end{array}$ & Arthralgia 57 (9\%) & $\begin{array}{l}\text { Diarrhea } 36(6 \%) \\
\text { Colitis } 26(4 \%) \\
\text { ALAT } 30(5 \%) \text {; ASAT } 22(4 \%) \\
\text { hypoT } 1(\leq 1 \%) \\
\text { Pruritus } 6(\leq 1 \%) \text {; Rash } 17(3 \%) \\
\text { Arthralgia } 1(\leq 1 \%)\end{array}$ & 1 (neutropenia) & NA \\
\hline $\begin{array}{l}\text { Brahmer et al., } \\
2015 \text { [9] }\end{array}$ & $\begin{array}{l}62 \\
82 \%\end{array}$ & $\begin{array}{l}\text { Diarrhea } 10 \\
(8 \%)\end{array}$ & 0 & НypoТ (4\%) & $6(5 \%)$ & Rash 5 (4\%) & $\begin{array}{l}\text { Anemia } 2(2 \%) \\
\text { Arthralgia } 7(5 \%) \\
\text { Myalgia } 2(2 \%) \\
\text { Nephritis }(3 \%) \\
\text { Neutropenia } 1(\leq 1 \%) \\
\text { NRP } 1(\leq 1 \%)\end{array}$ & $\begin{array}{l}\text { Diarrhea } 1(\leq 1 \%) \\
\text { PNP } 1(\leq 1 \%) \\
\text { Renal } 1(\leq 1 \%)\end{array}$ & 0 & $3 \%$ \\
\hline $\begin{array}{l}\text { Gettinger et al., } \\
2015[10]\end{array}$ & $\begin{array}{l}65 \\
61 \%\end{array}$ & $15(12 \%)$ & $6(5 \%)$ & $8(6 \%)$ & $9(7 \%)$ & $20(15 \%)$ & Nephritis $4(3 \%)$ & $\begin{array}{l}\text { Digestive } 1(\leq 1 \%) \\
\text { PNP } 3(2 \%) \\
\text { Hepatic } 1(\leq 1 \%)\end{array}$ & 3 (PNP) & $\begin{array}{l}\text { Yes } \\
\text { (grades 3-4) }\end{array}$ \\
\hline $\begin{array}{l}\text { McDermott et al., } \\
2015 \text { [11] }\end{array}$ & $\begin{array}{l}58 \\
76 \%\end{array}$ & $\begin{array}{l}\text { Diarrhea } 6 \\
(18 \%)\end{array}$ & $\begin{array}{l}\text { ASAT } 2(6 \%) \\
\text { ALAT } 4(12 \%)\end{array}$ & НуроТ 3 (9\%) & $1(3 \%)$ & $\begin{array}{l}\text { Rash } 9(26 \%) \\
\text { Pruritus } 6(18 \%)\end{array}$ & $\begin{array}{l}\text { Anemia } 2(6 \%) \\
\text { Arthralgia } 3(9 \%) \\
\text { NRP } 2 \text { (6\%) } \\
\text { Xerostomia } 2(6 \%)\end{array}$ & Pruritus $1(3 \%)$ & 0 & $\begin{array}{l}\text { Definitive } 5 \\
(15 \%) \\
\text { Temporary } 2 \\
(6 \%)\end{array}$ \\
\hline $\begin{array}{l}\text { Robert et al., } \\
2015 \text { [12] }\end{array}$ & $\begin{array}{l}64 \\
58 \%\end{array}$ & $\begin{array}{l}\text { Diarrhea } 33 \\
(16 \%)\end{array}$ & Frequent & NA & NA & $\begin{array}{l}\text { Pruritus } 35(17 \%) \\
\text { Rash } 31(15 \%) \\
\text { Vitiligo } 22(11 \%)\end{array}$ & ND & $\begin{array}{l}\text { Pruritus } 1(\leq 1 \%) \text {; rash } 1(\leq 1 \%) \\
\text { Diarrhea } 2(\leq 1 \%) \\
\text { ALAT } 2(\leq 1 \%)\end{array}$ & 0 & NA \\
\hline $\begin{array}{l}\text { Topalian et al., } \\
2014 \text { [13] }\end{array}$ & NA & $\begin{array}{l}\text { Diarrhea } 19 \\
(18 \%) \\
\text { Colitis } 2(2 \%)\end{array}$ & $\begin{array}{l}\text { ASAT } 4(4 \%) \\
\text { ALAT } 5(5 \%)\end{array}$ & $\begin{array}{l}\text { HypoT } 6(6 \%) \\
\text { Hypophysitis } 1 \\
(\leq 1 \%)\end{array}$ & $2(2 \%)$ & $\begin{array}{l}\text { Rash } 25(23 \%) \\
\text { Pruritus } 14(13 \%) \\
\text { Vitiligo } 10(9 \%)\end{array}$ & $\begin{array}{l}\text { Anemia } 5(5 \%) \\
\text { Arthralgia } 7(7 \%) \\
\text { Myalgia } 4(4 \%) \\
\text { Nephritis 3(3\%) } \\
\text { Thrombocytopenia } 5(5 \%) \\
\text { Uveitis1 }(\leq 1 \%) \\
\text { Xerostomia } 7(7 \%)\end{array}$ & $\begin{array}{l}\text { Diarrhea } 2(2 \%) \\
\text { Xerostomia } 1(\leq 1 \%) \\
\text { Anemia } 1(1 \%) \\
\text { Thrombocytopenia } 1(\leq 1 \%) \\
\text { hypoT } 1(\leq 1 \%)\end{array}$ & 0 & NA \\
\hline
\end{tabular}




\begin{tabular}{|c|c|c|c|c|c|c|c|c|c|c|}
\hline \multirow[t]{3}{*}{ Studies } & \multicolumn{10}{|l|}{ OASI } \\
\hline & \multirow{2}{*}{$\begin{array}{l}\text { Median age } \\
\text { Gender } \\
\text { Male (\%) }\end{array}$} & \multicolumn{6}{|c|}{ Type and incidence $n(\%)^{\mathrm{a}}$} & \multicolumn{3}{|l|}{ Severity } \\
\hline & & Digestive & Hepatic & Endocrine & PNP & Cutaneous & Others & Grade 3-4 & $\begin{array}{l}\text { Death related } \\
\text { to OASI }\end{array}$ & $\begin{array}{l}\text { IT } \\
\text { discontinuation }\end{array}$ \\
\hline $\begin{array}{l}\text { Ansell et al., } 2015 \\
\text { [14] }\end{array}$ & $\begin{array}{l}35 \\
52 \%\end{array}$ & $\begin{array}{l}\text { Diarrhea } 3 \\
(13 \%)\end{array}$ & Frequent & HypoT 2 (9\%) & NA & $\begin{array}{l}\text { Rash } 5(22 \%) \\
\text { Pruritus } 3(13 \%)\end{array}$ & $\begin{array}{l}\text { Pancreatitis } 1 \text { (4\%) } \\
\text { Thrombocytopenia } 4 \text { (17\%) }\end{array}$ & $\begin{array}{l}\text { Pancreatitis } 1(4 \%) \\
\text { Digestive } 2(9 \%) \\
\text { Thrombocytopenia 1 (4\%) }\end{array}$ & 0 & $9 \%$ \\
\hline $\begin{array}{l}\text { Motzer et al., } \\
2015 \text { [15] }\end{array}$ & $\begin{array}{l}61 \\
72 \%\end{array}$ & $\begin{array}{l}\text { Diarrhea } 16 \\
(10 \%)\end{array}$ & $\begin{array}{l}\text { ASAT } 8(5 \%) \\
\text { ALAT } 7(4 \%)\end{array}$ & НypoT $10(6 \%)$ & $8(5 \%)$ & $\begin{array}{l}\text { Pruritus } 17(10 \%) \\
\text { Rash } 16(10 \%)\end{array}$ & $\begin{array}{l}\text { Arthralgia } 13(8 \%) \\
\text { Nephritis } 2(\leq 1 \%)\end{array}$ & $\begin{array}{l}\text { Arthralgia } 1(\leq 1 \%) \\
\text { Pruritus } 1(\leq 1 \%) \\
\text { hypoT } 1(\leq 1 \%) \\
\text { ASAT } 2(\leq 1 \%) \text {; ALAT } 2(\leq 1 \%)\end{array}$ & 0 & $\begin{array}{l}\text { ASAT } 2(\leq 1 \%) \\
\text { Endocrine } 2 \\
(\leq 1 \%) \\
\text { Neurologic/ } \\
\text { Pulmonary } 2 \\
(\leq 1 \%)\end{array}$ \\
\hline $\begin{array}{l}\text { Le et al., } 2015 \\
\text { [16] }\end{array}$ & $\begin{array}{l}\text { NA } \\
58 \%\end{array}$ & $\begin{array}{l}\text { Diarrhea } 10 \\
(24 \%)\end{array}$ & ALAT 3 (7\%) & $\begin{array}{l}\text { HypoT/hypophysitis } \\
4(10 \%)\end{array}$ & $1(2 \%)$ & $\begin{array}{l}\text { Rash/pruritus } \\
10(24 \%)\end{array}$ & $\begin{array}{l}\text { Anemia } 8(20 \%) \\
\text { Arthralgia } 7(17 \%) \\
\text { Myalgia } 6(15 \%) \\
\text { Pancreatitis 6 (15\%) }\end{array}$ & $\begin{array}{l}\text { Anemia } 7(17 \%) \\
\text { Diarrhea } 2(5 \%) \\
\text { ALAT } 2(5 \%)\end{array}$ & 0 & NA \\
\hline $\begin{array}{l}\text { Postow et al., } \\
2015 \text { [17] }\end{array}$ & $\begin{array}{l}N+I \\
64 \\
66 \%\end{array}$ & $\begin{array}{l}\text { Diarrhea } 42 \\
(45 \%) \\
\text { Colitis } 22(23 \%)\end{array}$ & $\begin{array}{l}\text { ASAT } 20(21 \%) \\
\text { ALAT } 21(22 \%)\end{array}$ & $\begin{array}{l}\text { HypoT } 15(16 \%) \\
\text { hypophysitis } 11 \\
(12 \%)\end{array}$ & $\begin{array}{l}10 \\
(11 \%)\end{array}$ & $\begin{array}{l}\text { Rash } 57(60 \%) \\
\text { Pruritus } 33(35 \%) \\
\text { Vitiligo } 10(11 \%)\end{array}$ & $\begin{array}{l}\text { Arthralgia } 10(11 \%) \\
\text { Myalgia } 9(10 \%)\end{array}$ & $\begin{array}{l}\text { Diarrhea } 10 \text { (11\%); Colitis } 16 \\
(17 \%) \\
\text { Rash } 8 \text { (9\%); Pruritus } 1(\leq 1 \%) \\
\text { ALAT } 10(11 \%) \text {; ASAT } 7(7 \%) \\
\text { Hypophysitis 2 (2\%) } \\
\text { PNP } 2(2 \%)\end{array}$ & 1 (PNP) & NA \\
\hline $\begin{array}{l}\text { Robert et al., } \\
2014[2]\end{array}$ & $\begin{array}{l}59 \\
61 \%\end{array}$ & NA & NA & NA & NA & NA & NA & $\begin{array}{l}\text { Hepatitis } 1(\leq 1 \%) \\
\text { Rash } 1(\leq 1 \%) \\
\text { Pancreatitis } 1(\leq 1 \%) \\
\text { PNP } 1(\leq 1 \%) \\
\text { Diarrhea } 1(\leq 1 \%) \\
\text { Hypophysitis } 1(\leq 1 \%)\end{array}$ & 0 & $4(2 \%)$ \\
\hline $\begin{array}{c}\text { Robert et al., } \\
2015 \text { [18] }\end{array}$ & $\begin{array}{l}\mathrm{P} 2 \mathrm{~W} \\
61 \\
58 \% \\
\\
\mathrm{P} 3 \mathrm{~W} \\
63 \\
63 \%\end{array}$ & $\begin{array}{l}\text { Diarrhea } 87 \\
\text { (16\%) } \\
\text { Colitis } 15(3 \%)\end{array}$ & $8(\leq 1 \%)$ & $\begin{array}{l}\text { HypoT } 52(9 \%) \\
\text { hyperT } 27(5 \%) \\
\text { Hypophysitis } 3 \\
(\leq 1 \%) \\
\text { Diabetes } 2(\leq 1 \%)\end{array}$ & $6(\leq 1 \%)$ & $\begin{array}{l}\text { Rash } 78(14 \%) \\
\text { Pruritus } 79(14 \%) \\
\text { Vitiligo } 56(10 \%)\end{array}$ & $\begin{array}{l}\text { Arthralgia } 58(10 \%) \\
\text { Myositis2 }(\leq 1 \%) \\
\text { Nephritis } 1(\leq 1 \%) \\
\text { Uveitis4 }(\leq 1 \%)\end{array}$ & $\begin{array}{l}\text { Diarrhea } 10(2 \%) \text {; colitis } 11(2 \%) \\
\text { Arthralgia } 1(\leq 1 \%) \\
\text { HypoT } 1(\leq 1 \%) \\
\text { Hypophysitis } 2(\leq 1 \%) \text {; diabetes } \\
2(2 \%) \\
\text { Hepatitis } 8(\leq 1 \%) \\
\text { PNP } 1(\leq 1 \%)\end{array}$ & 0 & NA \\
\hline $\begin{array}{l}\text { Wolchok et al., } \\
2013 \text { [19] }\end{array}$ & $\begin{array}{l}N+I \\
58 \\
60 \% \\
\text { I then N } \\
64 \\
55 \%\end{array}$ & $\begin{array}{l}\text { Diarrhea } 18 \\
(34 \%) \\
\text { Colitis } 5(9 \%)\end{array}$ & $\begin{array}{l}\text { ASAT } 11(21 \%) \\
\text { ALAT } 11(21 \%)\end{array}$ & $\begin{array}{l}\text { HypoT } 2(4 \%) \\
\text { HyperT } 2(4 \%) \\
\text { Hypophysitis } 2 \\
(4 \%) \\
\text { Adrenal } 2(4 \%)\end{array}$ & $3(6 \%)$ & $\begin{array}{l}\text { Rash } 37(70 \%) \\
\text { Pruritus } 29(55 \%) \\
\text { Urticaria } 1(2 \%) \\
\text { Bullous } \\
\text { dermatosis1 (2\%) }\end{array}$ & nephritis 3 (6\%) & $\begin{array}{l}\text { PNP } 1(2 \%) \\
\text { hypophysitis } 1(2 \%) \\
\text { ASAT } 7(13 \%) \text {; ALAT } 6(11 \%) \\
\text { Diarrhea 3 (6\%); colitis } 2(4 \%) \\
\text { Nephritis 3 (6\%) } \\
\text { Rash } 2 \text { (4\%) }\end{array}$ & 0 & NA \\
\hline $\begin{array}{l}\text { Borghae et al., } \\
2015 \text { [20] }\end{array}$ & $\begin{array}{l}61 \\
52 \%\end{array}$ & $\begin{array}{l}\text { Diarrhea } 22 \\
(8 \%)\end{array}$ & $\begin{array}{l}\text { ASAT 3\% } \\
\text { ALAT 3\% }\end{array}$ & НуроТ (7\%) & $(3 \%)$ & $\begin{array}{l}\text { Rash }(9 \%) \\
\text { pruritus }(8 \%) \\
\text { erythema }(\leq 1 \%)\end{array}$ & $\begin{array}{l}\text { Anemia } 6(2 \%) \\
\text { Myalgia } 7(2 \%) \\
\text { Neutropenia } 1(\leq 1 \%)\end{array}$ & $\begin{array}{l}\text { Diarrhea } 2(\leq 1 \%) \\
\text { Myalgia } 1(\leq 1 \%) \\
\text { Anemia } 1(\leq 1 \%)\end{array}$ & 1 (encephalitis) & $\operatorname{PNP}(\leq 1 \%)$ \\
\hline
\end{tabular}

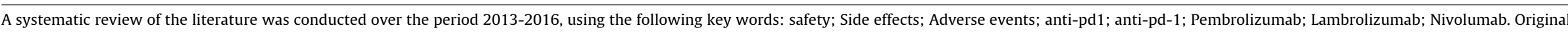

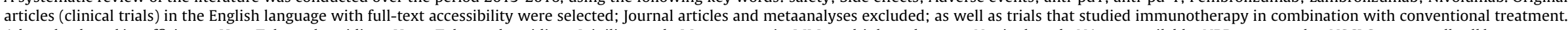

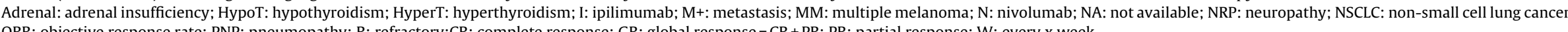

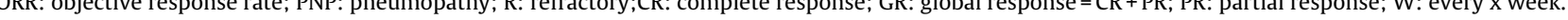

a The incidence of OASIs is provided with the number $(n)$ when available and/or\% of patients but in some trials only "frequent" was reported; Corresponding to an incidence> 3 to $5 \%$. 


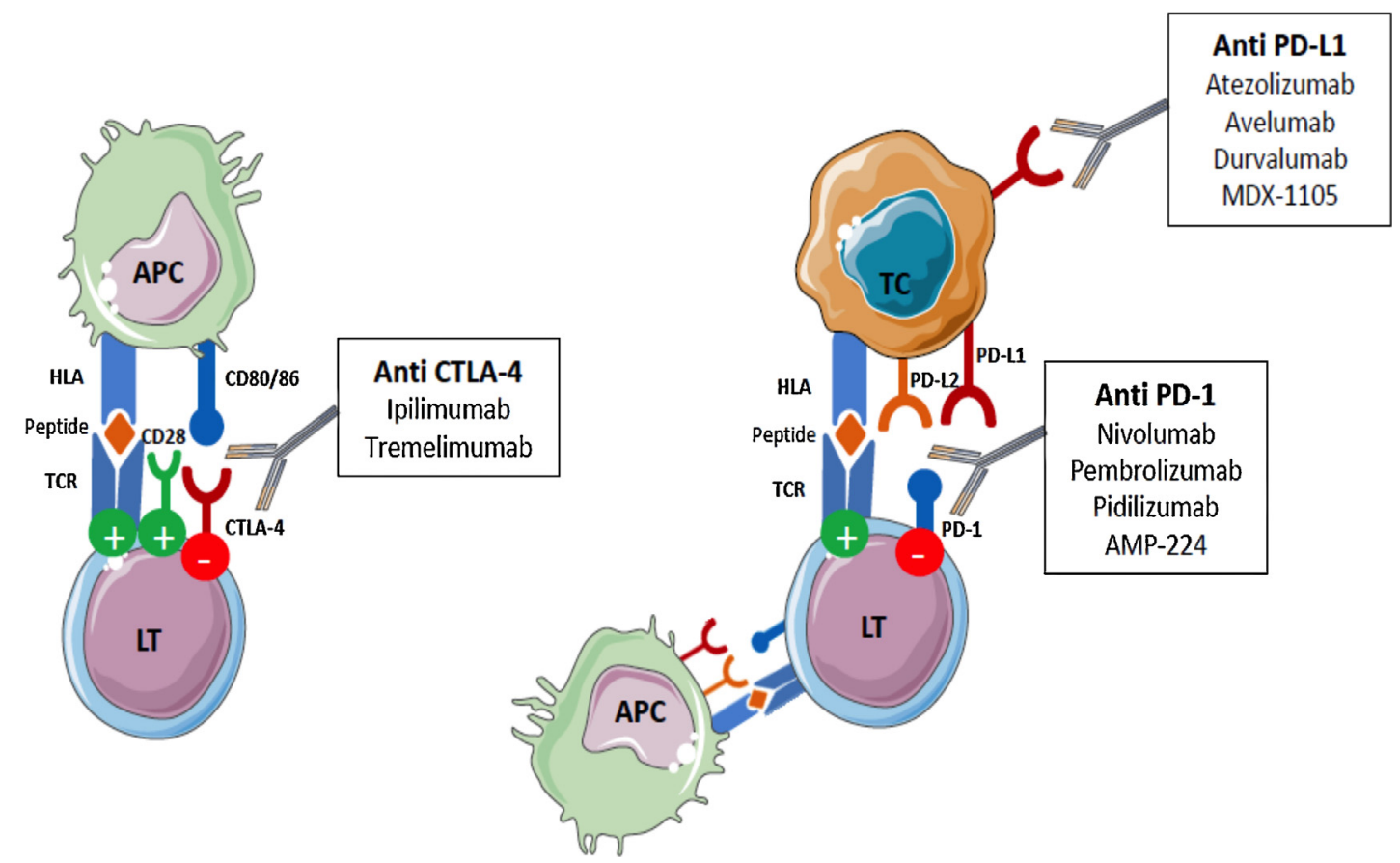

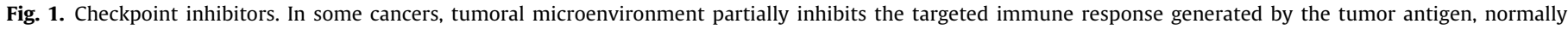

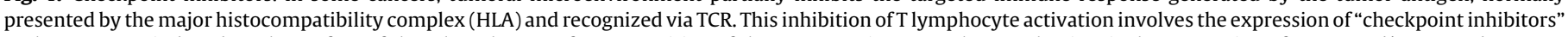

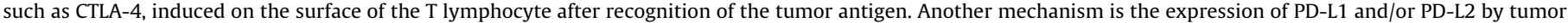

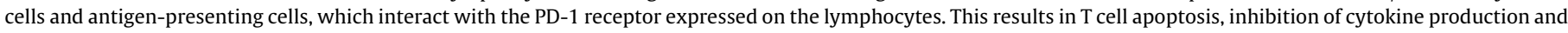

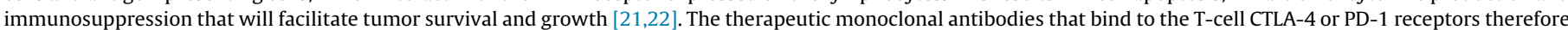

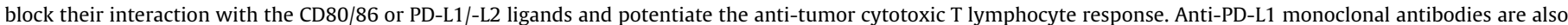

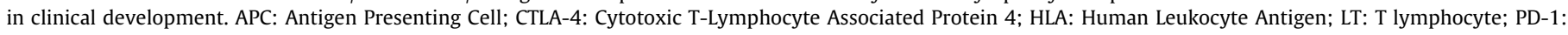
Programmed Death receptor; PD-L1/L2: Programmed Death-Ligand; TC: Tumoral Cell; TCR: T Cell Receptor.

by loss of peripheral tolerance. Recently, mechanisms of cancer immunotherapy have been developed in the journal and will not be discussed in detail (Fig. 1) [23]. The purpose of this article is to present an update on these opportunistic autoimmune manifestations secondary to cancer immunotherapy by specialists using immunotherapy for the treatment of severe autoimmune diseases.

\subsection{Terminology}

In literature, the side effects of immunotherapy have been reported with different terminology (e.g. adverse effects of special interest) but with a recent consensus on the term "ImmuneRelated Adverse Events" (irAEs) [24,25]. While using a common terminology makes sense, reported irAEs include both induced autoimmune manifestations and other "immunological" effects such as tumor progression or paradoxical worsening of opportunistic infections, similarly observed during an immune reconstitution syndrome. Therefore, identifying the manifestations of "Opportunistic Autoimmunity Secondary to Cancer Immunotherapy" (OASI) within irAEs will be worthwhile, in order to use a specific term referring to the pathogenesis of this new type of side effects.

\section{OASIs: reported autoimmune manifestations}

\subsection{Background}

The use of checkpoint inhibitors in trials and now in clinical practice gives us a more accurate picture of the broad spectrum of OASIs. Regarding the incidence rate, we must keep in mind biases related to exclusion of patients with pre-existing autoimmune diseases for clinical trials and the use of monotherapy in most trials. A systematic literature review was conducted to identify OASIs occurring with PD-1 pathway inhibitors (see
Methods, Tables 1 and 2) to complete the one with anti-CTLA4 recently published by our group [26]. OASIs reported during clinical trials mainly concerned gastrointestinal tract, skin, endocrine glands, liver and lung (Table 1 ), but can affect nearly all organs, as reported in "real life" series (out of trial setting).

Both systemic and organ-specific autoimmune manifestations have been reported. Recently, rare and various OASIs are being reported with the growing use of checkpoint inhibitors [27-44] (Table 2). Fortunately, occurrence of severe OASIs, mainly gastrointestinal and defined by grade 3 and 4 according to the Common Terminology Criteria for Adverse Events (CTCAE) is not common, especially for patients receiving anti-PD1 monotherapy $(\leq 10 \%)$. Indeed, OASIs are usually graded 1 or 2 according the CTCAE criteria, involving skin or digestive tract. While incidence of OASIs differed between trials, a recent meta-analysis provided better estimates of severe OASIs. For example, severe pneumonitis occurs in slightly less than $1 \%$ of patients [45]. Once again, these data have to be analyzed by taking into consideration the inclusion/exclusion criteria of the corresponding studies.

\subsection{OASIs by organs}

Dermatological OASIs are by far the most frequent OASIs in melanoma patients receiving checkpoint inhibitors. Vitiligo, erythema, psoriasis, bullous pemphigoid, DRESS syndrome and toxic epidermal necrolysis or Stevens-Johnson syndrome have been described [30,46-48]. Mucosal involvement with sicca syndrome has also been reported, especially with anti-PD-1, with sometimes positivity of antinuclear or La/SSB antibodies [43]. Gastrointestinal OASIs range from diarrhea (frequent with anti-CTLA-4) to inflammatory colitis mimicking both endoscopic and histological aspects of Crohn's disease. Pancreatitis, gastritis and celiac disease have also been reported. The endocrine OASIs observed with anti-PD-1 
Table 2

Case reports of OASIs with anti-PD1 (out of trials).

\begin{tabular}{|c|c|c|c|c|c|c|c|c|c|c|c|c|}
\hline Cases & $n$ & $\begin{array}{l}\text { Molecule+ } \\
\text { association }\end{array}$ & Cancer/stage & Age/gender & Organ involved & $\begin{array}{l}\text { Severity } \\
\text { NCICTCAE }\end{array}$ & $\begin{array}{l}\text { Delay of } \\
\text { onset }\end{array}$ & Risk Factor for AI & $\begin{array}{l}\text { Oncologic } \\
\text { response }\end{array}$ & Stop IT & $\begin{array}{l}\text { Treatment of the } \\
\text { OASI }\end{array}$ & $\begin{array}{l}\text { Evolution of } \\
\text { the OASI }\end{array}$ \\
\hline $\begin{array}{l}\text { Polat et Donofrio, } \\
2016 \text { [27] }\end{array}$ & 1 & $\mathrm{~N} 292 \mathrm{mg} / 2 \mathrm{~W}$ & NSCLC stage IV & $65 / \mathrm{M}$ & $\begin{array}{l}\text { Myasthenia Ab } \\
\text { anti-AChR - MuSK - }\end{array}$ & 3 & 10 days & NA & NA & Yes & Pyridostigmine & + \\
\hline Lau et al., 2016 [28] & 1 & $\mathrm{P}$ & MM M+ & 75/M & $\begin{array}{l}\text { Myasthenia Ab anti } \\
\text { AChR+ }\end{array}$ & 4 & 5 weeks & Myasthenia & Stability & No & NIV CT IVIg & + \\
\hline $\begin{array}{l}\text { Loochtan et al., } \\
2015 \text { [29] }\end{array}$ & 1 & $\mathrm{~N}+\mathrm{I}$ & SCLC & 70/M & $\begin{array}{l}\text { Myasthenia Ab } \\
\text { anti-AChR + }\end{array}$ & 5 & 16 days & NA & NA & Yes & CT PE IVIg & Death \\
\hline \multirow[t]{5}{*}{$\begin{array}{l}\text { Jour et al., } 2016 \\
\text { [30] }\end{array}$} & 5 & $\mathrm{~N} 3 \mathrm{mg} / \mathrm{kg}$ & $\begin{array}{l}\text { Tongue } \\
\text { Carcinoma } \mathrm{M}^{+}\end{array}$ & $63 / \mathrm{M}$ & Bullous Pemphigoïd & 2 & 96 days & 0 & NA & Yes & CT & + \\
\hline & & P $2 \mathrm{mg} / \mathrm{kg}$ & MM & 68/M & & 2 & 113 days & Psoriasis & TP & Yes & $\mathrm{CT}$ & + \\
\hline & & N 3 mg/kg/2W+I & $\begin{array}{l}\text { Urothelial } \\
\text { cancer } \mathrm{M}^{+}\end{array}$ & $74 / \mathrm{F}$ & & 2 & 112 days & 0 & PR & Yes & CT & + \\
\hline & & N 3 mg/kg & NSCLC & $73 / \mathrm{F}$ & & 2 & 49 days & 0 & TP & Yes & CT & - \\
\hline & & $\mathrm{N} 3 \mathrm{mg} / \mathrm{kg}$ & MM & 59/M & & 2 & 21 days & 0 & TP & Yes & CT & + \\
\hline $\begin{array}{l}\text { Vandiver et al., } \\
2016 \text { [31] }\end{array}$ & 1 & $\mathrm{~N} 3 \mathrm{mg} / \mathrm{kg}$ & MM M+ & $58 / \mathrm{F}$ & Nephritis & 3 & 4 days & Hypothyroidism & TP & Yes & CT & + \\
\hline $\begin{array}{l}\text { Hansen et al., } 2016 \\
\text { [32] }\end{array}$ & 1 & P $2 \mathrm{mg} / \mathrm{kg} / 3 \mathrm{~W}+\mathrm{I}^{*}$ & MM & $58 / \mathrm{M}$ & $\begin{array}{l}\text { Type } 1 \text { Diabetes Ab } \\
\text { anti-GAD + }\end{array}$ & 3 & 12 months & NA & PR & Yes & Insuline & + \\
\hline $\begin{array}{l}\text { Alabed et al., } 2015 \\
\text { [33] }\end{array}$ & 1 & $\mathrm{P}+\mathrm{I}^{*}$ & MM & $57 / \mathrm{M}$ & $\begin{array}{l}\text { Asymptomatic } \\
\text { pancreatitis }\end{array}$ & 2 & 3 cycles & $\begin{array}{l}\text { Previous colitis } \\
\text { with I }\end{array}$ & NA & NA & $\mathrm{NA}$ & NA \\
\hline $\begin{array}{l}\text { Danlos et al., } 2016 \\
\text { [34] }\end{array}$ & 1 & $\mathrm{~N} 3 \mathrm{mg} / \mathrm{kg} / 2 \mathrm{~W}$ & $\begin{array}{l}\text { Unresectable } \\
\text { MM }\end{array}$ & $57 / \mathrm{M}$ & $\begin{array}{l}\text { Cutaneous and } \\
\text { ganglionic } \\
\text { granulomatosis }\end{array}$ & 2 & 10 months & NA & CR & Yes & 0 & + \\
\hline $\begin{array}{l}\text { Koelzer et al., } 2016 \\
\text { [35] }\end{array}$ & 1 & $\begin{array}{l}\mathrm{N} \\
3 \mathrm{mg} / \mathrm{kg} / 2 \mathrm{~W}+\mathrm{I}^{*}\end{array}$ & MM & $35 / \mathrm{F}$ & $\begin{array}{l}\text { Pulmonary } \\
\text { granulomatosis, } \\
\text { meningitis, } \\
\text { myocarditis }\end{array}$ & 4 & 2 months & NA & TP & Yes & СT & - \\
\hline $\begin{array}{l}\text { Läubli et al., } 2015 \\
\text { [36] }\end{array}$ & 1 & P 2 mg $/ \mathrm{kg} / 3 \mathrm{~W}+\mathrm{I}^{*}$ & MM & $73 / \mathrm{F}$ & $\begin{array}{l}\text { Myocarditis with left } \\
\text { ventricular } \\
\text { dysfunction }\end{array}$ & 4 & 15 weeks & 0 & NA & Yes & CT & + \\
\hline \multirow[t]{2}{*}{$\begin{array}{l}\text { Garel et al., } 2016 \\
\text { [37] }\end{array}$} & 2 & P 2 mg $/ \mathrm{kg}$ & MM & $88 / \mathrm{F}$ & PMR & 3 & 1 day & NA & PR & Temporary & CT & + \\
\hline & & $\mathrm{P}$ & MM & 79/M & & 2 & 3 cycles & NA & PR & No & $\mathrm{CT}$ & + \\
\hline $\begin{array}{l}\text { Chan et al., } 2015 \\
\text { [38] }\end{array}$ & 2 & P $2 \mathrm{mg} / \mathrm{kg} / 3 \mathrm{~W}$ & MM M+ & $60 / \mathrm{M}$ & $\begin{array}{l}\text { Polyarthritis, } \\
\text { seronegative, } \\
\text { non-erosive }\end{array}$ & 2 & 14 months & 0 & $\mathrm{CR}$ & Temporary & SLZ & + \\
\hline
\end{tabular}


Table 2 (Continued)

\begin{tabular}{|c|c|c|c|c|c|c|c|c|c|c|c|c|}
\hline Cases & $n$ & $\begin{array}{l}\text { Molecule + } \\
\text { association }\end{array}$ & Cancer/stage & Age/gender & Organ involved & $\begin{array}{l}\text { Severity } \\
\text { NCICTCAE }\end{array}$ & $\begin{array}{l}\text { Delay of } \\
\text { onset }\end{array}$ & Risk Factor for AI & $\begin{array}{l}\text { Oncologic } \\
\text { response }\end{array}$ & Stop IT & $\begin{array}{l}\text { Treatment of the } \\
\text { OASI }\end{array}$ & $\begin{array}{l}\text { Evolution of } \\
\text { the OASI }\end{array}$ \\
\hline & & P $10 \mathrm{mg} / \mathrm{kg} / 3 \mathrm{~W}$ & MM M+ & $68 / \mathrm{F}$ & & 2 & 11 months & 0 & $\mathrm{CR}$ & Yes & NSAID HCQ & + partial \\
\hline $\begin{array}{l}\text { Salmon et al., } 2016 \\
\text { [39] }\end{array}$ & 1 & $\mathrm{P}$ & MM & $69 / \mathrm{M}$ & $\begin{array}{l}\text { Polyarthritis, } \\
\text { seronegative }\end{array}$ & 2 & NA & NA & NA & Temporary & СТ MTX & + \\
\hline $\begin{array}{l}\text { Nair et al., } 2016 \\
\text { [40] }\end{array}$ & 1 & $P+I^{*}$ & MM M+ & $52 / \mathrm{F}$ & $\begin{array}{l}\text { AlHA, } \\
\text { Erythroblastopenia }\end{array}$ & 4 & 3 cycles & $\begin{array}{l}\text { Previous } \\
\text { hepatitis with I }\end{array}$ & TP & NA & CT IVIg & + \\
\hline $\begin{array}{l}\text { Kong et al., } 2016 \\
\text { [41] }\end{array}$ & 1 & $\begin{array}{l}\mathrm{N} \\
3 \mathrm{mg} / \mathrm{kg} / 2 \mathrm{~W}+\mathrm{I}^{*}\end{array}$ & MM & $85 / \mathrm{M}$ & AIHA & 3 & 2 months & Coombs + & NA & Yes & CT & + \\
\hline $\begin{array}{l}\text { Kanameishi et al., } \\
2015 \text { [42] }\end{array}$ & 1 & $\mathrm{~N} 2 \mathrm{mg} / \mathrm{kg} / 3 \mathrm{~W}$ & MM & 79/F & $\begin{array}{l}\text { Immune } \\
\text { Thrombocytopenia } \\
\text { Ab anti-platelet + }\end{array}$ & 4 & 6 weeks & NA & NA & NA & $\begin{array}{l}\text { CT IVIg } \\
\text { romiplostim }\end{array}$ & + \\
\hline \multirow{12}{*}{$\begin{array}{l}\text { Cappelli et al., } 2016 \\
\text { [43] }\end{array}$} & 12 & $\mathrm{~N}$ & NSCLC & $61 / \mathrm{M}$ & Sjögren RF+ & 2 & 2 months & NA & Stability & No & Pilocarpine & + \\
\hline & & $N+\mathrm{I}$ & MM & $57 / \mathrm{M}$ & Sjögren & 2 & 2 months & NA & TP & No & CT cevimeline & - \\
\hline & & $\mathrm{N}$ & MM & $74 / \mathrm{F}$ & $\begin{array}{l}\text { Sjögren (parotitis) Ab } \\
\text { anti-SSB+ }\end{array}$ & 2 & 8 months & NA & PR & No & CT cevimeline & + \\
\hline & & $N+\mathrm{I}$ & $\begin{array}{l}\text { Renal cell } \\
\text { carcinoma }\end{array}$ & $58 / \mathrm{M}$ & $\begin{array}{l}\text { Polyarthritis, } \\
\text { seronegative }\end{array}$ & 3 & 12 months & $\begin{array}{l}\text { Previous colitis } \\
\text { with IT }\end{array}$ & Stability & Yes & $\begin{array}{l}\text { CT MTX } \\
\text { adalimumab }\end{array}$ & + \\
\hline & & $N+\mathrm{I}$ & $\mathrm{MM}$ & $46 / \mathrm{F}$ & $\begin{array}{l}\text { Polyarthritis, } \\
\text { seronegative, erosive }\end{array}$ & 3 & 13 months & $\begin{array}{l}\text { Previous colitis } \\
\text { with I }\end{array}$ & PR & Yes & $\begin{array}{l}\text { CT MTX } \\
\text { infliximab } \\
\text { etanercept }\end{array}$ & + \\
\hline & & $N+\mathrm{I}$ & NSCLC & $62 / \mathrm{M}$ & $\begin{array}{l}\text { Polyarthritis, } \\
\text { seronegative, erosive }\end{array}$ & 2 & 9 months & NA & Stability & Yes & $\mathrm{CT}$ & + partial \\
\hline & & $N+\mathrm{I}$ & MM & $35 / \mathrm{M}$ & $\begin{array}{l}\text { Polyarthritis, } \\
\text { seronegative }\end{array}$ & 3 & 3 months & $\begin{array}{l}\text { Previous colitis } \\
\text { with IT }\end{array}$ & Stability & No & CT adalimumab & + \\
\hline & & $\mathrm{N}$ & NSCLC & $56 / \mathrm{M}$ & $\begin{array}{l}\text { Polyarthritis Ab } \\
\text { anti-SSA+ }\end{array}$ & 2 & 2 months & NA & Stability & No & CT & + \\
\hline & & $N+\mathrm{I}$ & MM & $66 / \mathrm{M}$ & $\begin{array}{l}\text { Polyarthritis, } \\
\text { seronegative }\end{array}$ & 3 & 23 months & $\begin{array}{l}\text { Previous colitis } \\
\text { with IT }\end{array}$ & PR & No & $\begin{array}{l}\text { CT infliximab } \\
\text { adalimumab }\end{array}$ & + \\
\hline & & $N+\mathrm{I}$ & SCLC & $57 / \mathrm{M}$ & $\begin{array}{l}\text { Spondyloarthritis } \\
\text { HLAB27- }\end{array}$ & 2 & 8 months & NA & PR & No & CT colchicine & + \\
\hline & & $N+\mathrm{I}$ & NSCLC & $42 / \mathrm{M}$ & $\begin{array}{l}\text { Polyarthritis, } \\
\text { seronegative }\end{array}$ & 2 & 3 months & NA & PR & No & $\begin{array}{l}\text { Dexamethasone } \\
(\mathrm{M}+\text { cerebral })\end{array}$ & + \\
\hline & & $\mathrm{N}$ & NSCLC & $75 / \mathrm{F}$ & $\begin{array}{l}\text { Polyarthritis, } \\
\text { seronegative }\end{array}$ & NA & 5 months & NA & PR & No & NSAID & NA \\
\hline $\begin{array}{l}\text { Ishikawa et Oashi, } \\
2016 \text { [44] }\end{array}$ & 1 & $\mathrm{~N} 2 \mathrm{mg} / \mathrm{kg} / 3 \mathrm{~W}$ & Advanced MM & $55 / \mathrm{M}$ & Hypophysitis & 3 & 84 days & NA & Stability & No & Hydrocortisone & + \\
\hline
\end{tabular}

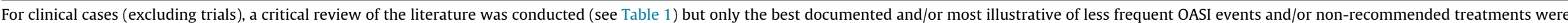

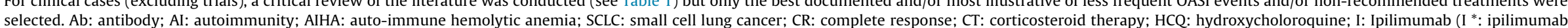

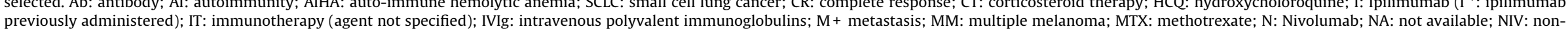

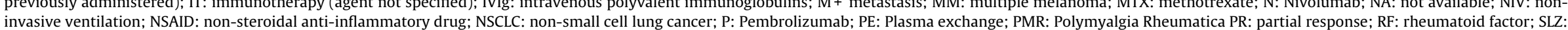
salazopirin; TP: tumor progression; NCICTCAE: common terminology criteria for adverse events. 
affect mainly the thyroid gland with hypothyroidism or, less frequently, hyperthyroidism with usually a spontaneous regression or secondary hypothyroidism. Hypophysitis, (commonly with antiCTLA-4, rarely reversible [49] and mainly associated with central hypothyroidism), adrenal insufficiency or type 1 diabetes can also occur $[32,50,51]$. Pulmonary OASIs are represented by inflammatory lung diseases, including sarcoidosis and BOOPs, with various radiological and histological aspects [52], and more rarely pleural effusions. Finally, hepatic OASIs are mostly biological (elevated transaminases) but authentic autoimmune hepatitis are possible, requiring histological documentation.

Less frequent OASIs have also been reported (Table 2), including neurological (peripheral neuropathy, aseptic meningitis, Guillain-Barre syndrome, myelitis, encephalitis, myasthenia gravis), renal (lupus-like glomerulonephritis, granulomatous interstitial nephritis), hematological (hemolytic anemia, thrombocytopenia, neutropenia, aplastic anemia, acquired hemophilia), articular (giant-cell arteritis (GCA)/polymyalgia rheumatica (PMR), lupus, rheumatoid arthritis (RA) (which one treatment option is based on a selective CD28/CD80-86 blockade via CTLA4 molecule), muscular (myositis), cardiac (pericarditis, myocarditis) and ophthalmological (episcleritis, uveitis, retinitis) manifestations.

\section{OASIs: risk factors and diagnostic workup}

\subsection{Risk factors}

To date, there is no predictive biomarker (clinical or biological) for OASIs occurrence. However, the optimal management of OASIs is based on their early recognition and some clinical parameters might help to optimize this monitoring. OASIs occur mostly 3 to 6 months after treatment initiation [53]. The median delay also varies according to OASIs nature: dermatological (5 weeks), digestive ( 7 weeks), hepatic ( 8 weeks), pulmonary ( 9 weeks) or endocrine ( 10 weeks). However, they can occur after a longer period (sometimes more than a year), and even after treatment discontinuation, highlighting the need for prolonged monitoring. Some OASIs seem more specific to the type of cancer, such as vitiligo and melanoma [54], or to the type of molecule (anti CTLA-4 versus PD-1) [55]. Patients receiving combination therapy develop more OASIs than those treated with monotherapy [3]. While a dose effect has been suggested with anti-CTLA-4 [26], no cumulative toxicity has been observed with anti-PD-1 [8]. Finally, identification of patients at highest risk of autoimmunity is important, based on personal and/or familial history of autoimmunity or ongoing active autoimmune disease, but other characteristics such as age or presence of renal/hepatic impairment do not seem to favor OASIs occurrence. So far, screening for OASIs occurrence should be offered similarly to all treated patients.

\subsection{Diagnostic workup}

In theory, OASIs presentation can mimic any known autoimmune disease (see above). Therefore, the purpose is not to list exhaustively all the clinical manifestations (Tables 1 and 2) or the corresponding diagnostic tests which are well known by internists and other organ specialists, but rather to point out the specificities of OASIs as compared to corresponding autoimmune diseases (Table 3). Indeed, autoantibodies are often missing in OASIs, challenging the differential diagnostic approach (cancer evolution, opportunistic and nosocomial infections, thrombosis.). Moreover, there is no specific radiological presentation and sometimes biopsy is required [56,57]. Referring patients with OASI to an organ specialist or internist has been recommended but only for severe OASIs (grades 3 and 4) [25]. Ideally, we consider that these specialists should be involved also for mild and moderate OASIs when possible, first because these OASIs can progress to more severe manifestations, and second to allow the different specialists in each center to become familiar and gain experience with the entire spectrum of OASIs.

\section{OASIs: treatment}

\subsection{Pre-treatment assessment}

The optimal management of OASIs depends on pre-treatment evaluation. This assessment has two aims: to identify risk factors of autoimmunity and to establish baseline (or reference) clinical and paraclinical evaluation before treatment initiation in order to facilitate the distinction between some abnormalities induced by the treatment and those that were pre-existing. The questioning should seek to identify personal or familial history of autoimmunity and suggestive symptoms (e.g. joint pain, digestive disorders), which will be a reference when monitoring treatment. Similarly, laboratory tests including liver, renal, endocrine, as well as electrocardiogram and chest imaging are essential. A pre-immunotherapy workup has been proposed by some expert cancer centers [25]. Apart from screening for thyroid dysfunction, common in the general population, the value of other hormonal measurements before or during follow-up is questionable in the absence of suggestive symptoms. The search for antinuclear antibodies, except for defining a reference value, has no impact on treatment decision. So far, no data demonstrated that their presence could identify a group at higher risk of OASIs. Conversely, it seems interesting to associate this pre-therapeutic assessment with the one proposed before initiation of anti-TNF, since some of these patients may require this biologic agent in case of severe OASIs. In this respect, we propose a minimum pre-therapeutic assessment (Table 4), while recognizing the value of collecting additional parameters in a biological resource center as part of research programs assessing predictive biomarkers.

\subsection{Patients with previous autoimmune condition}

Pre-therapeutic assessment is even more important for patients already suffering from an autoimmune disease. Owing to a theoretical risk of worsening of the pre-existing autoimmune disease, these patients have been excluded from clinical trials and there are few safety data with checkpoint inhibitors in such patients. Some case reports have been published regarding patients with pre-existing RA, ulcerative colitis (UC), or Behçet's disease successfully treated with ipilimumab (anti CTLA-4) [58]. No worsening of the autoimmune condition was observed and the anticancer effect of immunotherapy was preserved. Conversely, severe relapses of multiple sclerosis or UC were observed in two patients treated with ipilimumab, while improving the outcome of their metastatic melanoma $[59,60]$. So far, the largest retrospective series of patients with pre-existing autoimmune diseases treated with ipilimumab $(n=30)$ revealed that $50 \%$ of patients developed either OASIs or exacerbation of the pre-existing autoimmune disease, which were usually controlled with corticosteroids or increased immunomodulatory treatments [61]. The occurrence of severe OASIs seems less common with the anti-PD-1 in clinical trials comparing pembrolizumab or nivolumab versus ipilimumab in melanoma patients $[3,18]$. Therefore, anti-PD-1 should probably be preferred for patients with pre-existing autoimmune disease. Recently, pembrolizumab was prescribed successfully in two patients with metastatic melanoma with active autoimmune disease, namely pulmonary vasculitis (Churg-Strauss) and bullous pemphigoid $[48,62]$. Thus, currently available data do not suggest that a 
Table 3

OASIs: diagnostic and therapeutic aspects.

\begin{tabular}{|c|c|c|c|c|c|}
\hline Manifestations & Management & & & & \\
\hline & $\begin{array}{l}\text { Severity } \\
\text { Place } \\
\text { Immunotherapy } \\
\text { Diagnostic tools }\end{array}$ & $\begin{array}{l}\text { Grade } 1 \\
\text { Outpatient } \\
\text { Continuation }\end{array}$ & $\begin{array}{l}\text { Grade } 2 \\
\text { Outpatient } \\
\text { Temporary stop }\end{array}$ & $\begin{array}{l}\text { Grade } 3 \\
\text { Hospitalisation } \\
\text { Stop } \pm \text { definitive }^{\text {a }}\end{array}$ & $\begin{array}{l}\text { Grade } 4 \\
\text { Hospitalisation }\end{array}$ \\
\hline $\begin{array}{l}\text { Gastrointestinal } \\
\text { Diarrhea } \\
\text { Colitis ("Crohn like") }\end{array}$ & Stool Calprotectin & $\begin{array}{l}\text { Diet + Loperamide } \\
\text { Budesonide } \\
<4 \text { stools/d }\end{array}$ & $\begin{array}{l}\text { Colonoscopy? } \\
\text { CT oral } 0.5-1 \mathrm{mg} / \mathrm{kg}^{\mathrm{b}} \\
4-6 \text { stools/d }\end{array}$ & \multicolumn{2}{|l|}{$\begin{array}{l}\text { Scanner: perforation? Colonoscopy ++ } \\
\text { CT } 1-2 \mathrm{mg} / \mathrm{kg} \text { iv then at D3 CT } 1 \mathrm{mg} / \mathrm{kg} \\
\text { CTR: Infliximab } 5 \mathrm{mg} / \mathrm{kg} \text { (/2 weeks) } \\
\text { CTD: AZA } \\
>6 \text { stools/d, ileus, peritoneal irritation }\end{array}$} \\
\hline \multirow{3}{*}{$\begin{array}{l}\text { Dermatologic } \\
\text { Rash (lupus) } \\
\text { Dryness (Sjögren) }\end{array}$} & Auto-Abs often missing & $\begin{array}{l}\text { Transaminases }<2.5 \mathrm{~N} \\
\text { And/or Bilirubin }<1.5 \mathrm{~N}\end{array}$ & $\begin{array}{l}\text { CT oral } 0.5-1 \mathrm{mg} / \mathrm{kg}^{\mathrm{b}} \\
\text { Transaminases }>2.5 \mathrm{~N} \\
\text { and } / \text { or Bilirubin }>1.5 \mathrm{~N}\end{array}$ & $\begin{array}{l}\text { CT } 1-2 \mathrm{mg} / \mathrm{kg} \text { then at D3 CT } 1 \mathrm{mg} / \mathrm{kg} \\
\text { CTD: AZA, MMF }(500 \mathrm{mg} \times 2 / \mathrm{d}) \\
\text { Transaminases }>5 \mathrm{~N} \text { and/or } \\
\text { Bilirubin }>3 \mathrm{~N}\end{array}$ & \\
\hline & $\begin{array}{l}\text { Histology (skin, } \\
\text { salivary gland) } \\
\text { Complement } \\
\text { Proteinuria }\end{array}$ & $\begin{array}{l}\text { Anti-histaminics } \\
\text { Dermocortisone }\end{array}$ & $\begin{array}{l}\mathrm{CT} \text { oral } 0.5-1 \mathrm{mg} / \mathrm{kg}^{\mathrm{b}} \\
\pm \mathrm{HCQ}\end{array}$ & $\begin{array}{l}\text { CT } 1-2 \mathrm{mg} / \mathrm{kg} \text { then at D3 CT } 1 \mathrm{mg} / \mathrm{kg} \\
\text { CTD: AZA, MMF } \pm \mathrm{HCQ}\end{array}$ & \\
\hline & & $<10 \%$ BS & $10-30 \%$ BS & > 30\% BS, surinfections & \\
\hline Articular & $\begin{array}{l}\text { Lupus, RA, Sjögren, } \\
\text { GCA/PMR, ANCA. }\end{array}$ & Analgesics & $\begin{array}{l}\text { CT oral } 0.2-0.5 \mathrm{mg} / \mathrm{kg}^{\mathrm{b}} \\
\text { HCQ }\end{array}$ & $\begin{array}{l}\text { CT } 0.5-1 \mathrm{mg} / \mathrm{kg} \\
\text { CTD: MTX, AZA, anti-TNF }\end{array}$ & \\
\hline Pulmonary & $\begin{array}{l}\text { Sarcoidosis } \\
\text { BOOP }\end{array}$ & - & $\begin{array}{l}\text { BAL? } \\
\text { CT oral } 0.5-1 \mathrm{mg} / \mathrm{kg}^{\mathrm{b}} \\
\text { Dyspnea }\end{array}$ & $\begin{array}{l}\text { CT } 1-2 \mathrm{mg} / \mathrm{kg} \text { iv then at D3 CT } 1 \mathrm{mg} / \mathrm{kg} \\
\text { CTR: Infliximab } 5 \mathrm{mg} / \mathrm{kg} \text { (/2 weeks) } \\
\text { Oxygenotherapy }\end{array}$ & Intubation \\
\hline $\begin{array}{l}\text { Endocrine } \\
\text { Thyroiditis } \\
\text { Hypophysitis }\end{array}$ & $\begin{array}{l}\text { Hypothyroidism +++ } \\
\text { Hyperthyroidism } \\
\text { hypophysary MRIOther } \\
\text { hormones }\end{array}$ & $\begin{array}{l}\text { Hormonotherapy } \pm \mathrm{CT} \text { oral } \\
1 \mathrm{mg} / \mathrm{kg} \\
\text { Treatment of } \\
\text { hyperthyroidism } \pm \mathrm{CT} \\
1 \mathrm{mg} / \mathrm{kg}\end{array}$ & & \multicolumn{2}{|c|}{$\begin{array}{l}\text { CT } 1 \mathrm{mg} / \mathrm{kg}+\text { Hormonotherapy then at } 4 \text { weeks, } \\
\text { hydrocortisone } 30 \mathrm{mg} / \mathrm{j}\end{array}$} \\
\hline
\end{tabular}

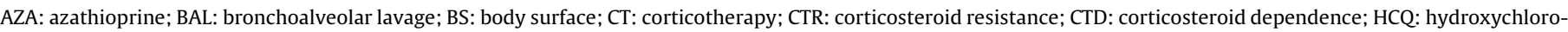

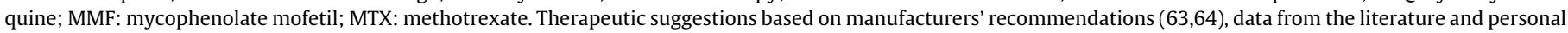
experience of the authors that can be adapted on a case-by-case basis in the absence of prospective data.

a Non-mandatory stop for immunotherapy if endocrinopathy, but definitive stop in case of recurrent grade 3 or grade 4 .

b CT after 1 week, if no improvement after discontinuation of immunotherapy.

Table 4

Pretreatment workup and biological monitoring under immunotherapy.

\begin{tabular}{|c|c|c|c|}
\hline Before treatment & Specificities & During treatment ${ }^{\mathrm{a}}$ & After treatment ${ }^{\mathrm{a}}$ \\
\hline Complete Blood Count ${ }^{\mathrm{b}}$ & & + & /3 months \\
\hline Plasmatic proteins electrophoresis ${ }^{\mathrm{b}}$ & & NS & NS \\
\hline Liver tests $^{\mathrm{b}}$ & & + & /3 months \\
\hline Serologies HBV, HCV, HIV ${ }^{\mathrm{b}}$ & & NS & NS \\
\hline $\begin{array}{l}\text { Anti-nuclear antibodies } \pm \text { anti-dsDNA } \\
\text { and anti-nuclear antigens } s^{\mathrm{b}, \mathrm{c}}\end{array}$ & & NS & NS \\
\hline Chest $\mathrm{X}$ ray ${ }^{\mathrm{b}}$ & $\begin{array}{l}\text { A CT scan is often available } \\
\text { during neoplasia } \\
\text { evaluation }\end{array}$ & NS & NS \\
\hline Tuberculosis test ${ }^{\mathrm{b}}$ & & NS & NS \\
\hline TSH, T4, anti-thyroid antibodies ${ }^{\mathrm{d}}$ & $\begin{array}{l}\text { Other hormonal testing can } \\
\text { be proposed }\end{array}$ & /1 to 2 months & 13 months \\
\hline C-reactive protein ${ }^{\mathrm{d}}$ & & + & NS \\
\hline Blood glucose $^{\mathrm{d}}$ & & + & /3 months \\
\hline Coagulation tests ${ }^{\mathrm{d}}$ & & + & 3 months \\
\hline $\begin{array}{l}\mathrm{Na}, \mathrm{K}, \mathrm{HCO} 3, \mathrm{Ca} \text {, serum creatinin, } \\
\text { urinary labstick or sample } \\
\text { proteinuria/creatininuria ratio }^{\mathrm{d}}\end{array}$ & & $+/ 1$ to 2 months & /3 months \\
\hline
\end{tabular}

+: at each infusion; NS: no systematic reevaluation.

a After treatment initiation, clinical and biological monitoring focuses on the most frequent OASIs, “A.B.C.D.E." Asat-alat; Breath (Bronchopulmonary); Cutaneous; Digestive (Diarrhea); Endocrine manifestations.

b Some patients receiving immunotherapy will develop an OASI and/or relapse of a pre-existingautoimmune disease. They are likely to receive corticosteroid therapy and in some cases, anti-TNF biotherapy. It seems advisable to carry out a pre-therapeutic assessment inspired of the one carried out before anti-TNF treatment.

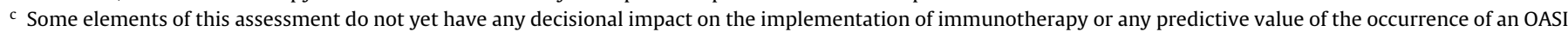
and are therefore debatable or carried out for research purposes.

d Finally, other parameters are specific to the OASIs encountered under immunotherapy. 
pre-existing autoimmune disease should be a systematic exclusion criteria for immunotherapy but estimation of the benefit/risk ratio should be carefully discussed with the patient and the oncologist. Combination therapy should probably be avoided in first line therapy for such patients.

\subsection{Treatment of OASIs}

The optimal management is based on the earliest possible identification of OASI. The therapeutic challenge is to manage opportunistic autoimmunity while preserving antitumor activity of immunotherapy. There are no prospective studies evaluating the treatment modalities of OASIs. Some recommendations developed by the manufacturers $[63,64]$ as well as by experts $[65,66]$ are available. These resources also provide for each organ the precise definition of OASIs severity based on CTCAE criteria. Indeed, this severity determines the nature and intensity of immunomodulatory treatments to be initiated and the decision to resume (or not) immunotherapy [67]. Overall, grade 1 OASIs are treated symptomatically and do not require discontinuation of immunotherapy. For grade 2 OASIs (moderate toxicity), oral corticosteroids ( 0.5 to $1 \mathrm{mg} / \mathrm{kg}$ ) may be proposed if the OASI persists after one week of symptomatic treatment and immunotherapy discontinuation. Finally, for grades 3 or 4 OASIs (severe or life-threatening toxicity), the permanent immunotherapy discontinuation is discussed (except for skin and endocrine OASIs), and treatment with high dose of intravenous corticosteroids $(\geq 1-2 \mathrm{mg} / \mathrm{kg}$ ) is initiated. Patients sensitive to corticosteroids often respond within few days. In this case, after OASI improvement to an equivalent of grade 1 , corticosteroid therapy is gradually tapered over one month. Conversely, in case of non-response or worsening, an immunomodulatory treatment with anti-TNF or other immunosuppressant should be initiated according to the affected organ (Table 3). There is no data showing that patients who received such treatments for OASI have a lower tumor response [68,69]. However, the long-term impact of biological or other immunosuppressive drugs on cancer outcome has to be more thoroughly investigated. The decision of outpatient or inpatient treatment (sometimes intensive care unit required) depends on OASI severity together with patient comorbidities and/or the need to rule out a differential diagnosis (e.g. infection, neoplasia progression). Like the need of strong immunosuppressive therapy in the setting of conventional autoimmune pathologies, the issue of opportunistic infections prophylaxis arises. For Pneumocystis carinii pneumonia (PCP), prophylactic sulfamethoxazole-trimethoprim administration should be discussed considering past anti-cancer treatments. Some groups suggest systematic prophylaxis but these recommendations are not evidence-based and may still allow a more individualized approach to avoid sulfamethoxazole-trimethoprim toxicities (cutaneous, digestive, hematological). Some oncologists rely on US guidelines suggesting prophylaxis (sulfamethoxazole trimethoprim-, atovaquone or pentamidine) for patients treated with corticosteroids $>20 \mathrm{mg} /$ day for 4 weeks [70,71]. Corticosteroid treatment should be as short as possible and in regard to endocrine manifestations may even be questionable owing to the effectiveness of hormone replacement therapy and the very low probability that steroids will avoid the use of hormone therapy. There are no recommendations for fungal or viral prophylaxis. TNF antagonists should be reserved for severe manifestations but they have the advantage of faster immunosuppressive action. Infliximab is initiated at $5 \mathrm{mg} / \mathrm{kg}$ in case of no improvement, sometimes after only 3 days of intravenous high-dose corticosteroids, and a second dose of $5 \mathrm{mg} / \mathrm{kg}$ may be administered after 2 weeks if necessary. For some OASIs of mild/moderate severity and for which manifestations suggest a potential benefit of hydroxychloroquine (HCQ) in addition to corticosteroids (e.g. arthralgia, arthritis), such association can be proposed, especially with the "favorable" benefit/risk profile of HCQ from a neoplastic perspective [72]. Regarding the resuming of immunotherapy, it is conditioned by the improvement of severe manifestations to grade 1 or less, a significant decrease of corticosteroids ( $<10 \mathrm{mg} /$ day) and the absence of permanent immunosuppression. In practice, some patients have been retreated after severe pneumonia without relapses, while, more rarely, some patients relapsed when corticosteroids were stopped even if immunotherapy had not been resumed [45,52]. Preliminary data suggest that a patient who developed OASI under anti-CTLA-4 can be treated with anti-PD-1 without occurrence of a new OASI [73].

\section{OASIs: perspectives}

\subsection{New therapeutic approaches}

Despite the sustainable tumor response observed with checkpoint inhibitors, a significant proportion of patients do not respond to these treatments. Several new strategies should allow better clinical outcomes in these non-responder patients. Combined nivolumab (anti-PD-1) and ipilimumab (anti-CTLA-4) showed a higher response rate and more sustained response than either agent alone. However, such a combination also tends to increase the OASIs severity, especially diarrhea, colitis and hepatitis. In the landmark study, the need for immunomodulatory agents was reported for $83 \%$ of patients with OASIs in the combination group versus only half of patients treated with ipilimumab or nivolumab alone [3]. Most of these OASIs were manageable and no manifestation seemed specifically related to the combination strategy. The combination of a checkpoint inhibitor with conventional chemotherapy or targeted therapy such as BRAF, MEK or other small molecule inhibitors is also being studied [74-77]. Radiation therapy is another attractive synergistic, by inducing multiple immunomodulatory effects on both the tumor and its microenvironment [78].

Finally, new immunomodulatory monoclonal antibodies are being developed to overcome the resistance to the first generation of checkpoint inhibitors. Of note, promising preclinical anti-OX40 antibody agonists, which activate the costimulatory signal on $\mathrm{T}$ cells, and are being tested in early-stage clinical trials [79]. Some of these new therapeutic strategies will likely be validated in the near future for a wide range of cancers, with the common goal of enhancing the anti-tumor immunity but thereby the occurrence of OASIs. There are potentially so many combinations to test that using mathematical modelling tools will be valuable to guide the selection and timing of future combinations [80].

\subsection{Predictive biomarkers for OASIs}

Given that a significant proportion of patients receiving immunotherapy will develop OASI, research on the identification of predictive biomarkers of severe autoimmune toxicity is needed. [81] Indeed, early identification of patients who may be predisposed to develop OASIs could promote their early detection or even the development of preventive strategies. To date, most studies have focused on tumor biomarkers predictive of treatment response [82]. Regarding OASI biomarkers, some studies assessed the genetic variants of CTLA-4. Some variants appear to influence treatment response with improved overall survival and without evidence of autoimmunity [83,84]. PD-1 and CTLA-4 polymorphisms are also associated with various autoimmune diseases such as RA, lupus, diabetes, thyroiditis [85]. Some OASIs clearly share the clinical features of these diseases and further 
research should focus on these possible genetic predisposition [86]. Recent findings also showed that microbiota-associated biomarkers correlated with lower occurrence of anti-CTLA-4induced colitis [87]. An increased proportion of Bacteroidetes phylum was found in the patients "resistant" to the onset of colitis, possibly related to an immunomodulatory role of these bacteria through stimulation of Treg differentiation. If validated in larger series, this data could help to identify a subgroup of patients at higher or lower risk of colitis, with the possible evaluation of preventive interventions. Other biological markers associated with the occurrence of OASIs, especially digestive, have been identified in peripheral blood such as increased eosinophils [88,89], IL-17 [90], CD177 expression and CEACAM1 genes and a high infiltration of the lamina propria by neutrophils in the colon during treatment [91]. Another group assessed blood transcriptome of patients with melanoma and identified differential gene expression in patients prone to develop gastrointestinal OASIs [92]. Finally, some studies correlated the immune tumor phenotype with treatment response, but these data were not used in regard to OASIs occurrence.

\subsection{Registries and multidisciplinary approach}

The spectrum of OASIs is extremely broad and its incidence is probably higher than suspected clinically. An autopsy study of a patient with melanoma treated with ipilimumab and nivolumab identified asymptomatic lymphocytic myocarditis, and inflammatory lesions of the central nervous system, liver and bone marrow [35]. This observation highlights for the first time the potential presence of sub-clinical and diffuse inflammation induced by these agents. Thus, physicians of all specialties can be confronted to OASIs. Oncologists are encouraged to develop a local network with organ specialists trained to manage OASIs, as a collaborative approach will help to reduce morbidity and to guide therapeutic interruptions [25]. Multidisciplinary meetings dedicated to OASIs management might be developed in the future. Immunotherapy including the use of the checkpoint inhibitors is now entering routine practice and the number of patients developing OASIs will rapidly increase. National registries set up to collect cases of malignancies and opportunistic infections in patients receiving biologics in RA and other autoimmune diseases could be an example to follow [93]. Moreover, the establishment of a biological collection/database seems essential. This would provide additional real life data to those from clinical trials and can be the basis for translational research. In addition, long-term monitoring of these patients would answer some clinical questions, such as the impact of immunosuppressive drugs/biologics on cancer and preexisting autoimmune disease evolution under immunotherapy. In France, the national pharmacovigilance registry Registry of Severe Adverse Effects of Monoclonal Antibodies in Cancer Immunomodulators (REISAMIC) is now available to report serious adverse events related to immunotherapy [25].

\section{Conclusion}

OASIs are relatively common, usually benign and easily managed, therefore not compromising the oncologic benefit obtained for some patients with extensive and/or refractory neoplasms. However, potential severity justifies an early OASIs detection, which can be facilitated by a pre-treatment workup and a close/systematic clinical and laboratory monitoring. Collaboration between oncologists, internists and organ specialists for the diagnosis and treatment of these induced autoimmune manifestations is worthwhile. Development of validated therapeutic algorithms and predictive biomarkers for the onset of OASIs represents future research priorities.

\section{Disclosure of interest}

The authors have not supplied their declaration of competing interest.

\section{Appendix A. Supplementary data}

Supplementary data associated with this article can be found, in the online version, at http://dx.doi.org/10.1016/j.revmed. 2017.01.004.

\section{References}

[1] Robert C, Thomas L, Bondarenko I, O’Day S, Weber J, Garbe C, et al. Ipilimumab plus dacarbazine for previously untreated metastatic melanoma. N Engl J Med 2011;364:2517.

[2] Robert C, Ribas A, Wolchok JD, Hodi FS, Hamid O, Kefford R, et al. Anti-programmed-death-receptor-1 treatment with pembrolizumab in ipilimumab-refractory advanced melanoma: a randomised dose-comparison cohort of a phase 1 trial. Lancet 2014:384:1109-17.

[3] Larkin J, Chiarion-Sileni V, Gonzalez R, Grob JJ, Cowey CL, Lao CD, et al. Combined Nivolumab and Ipilimumab or monotherapy in untreated melanoma. N Engl J Med 2015;373:23-34.

[4] Hamid O, Robert C, Daud A, Hodi FS, Hwu WJ, Kefford R, et al. Safety and tumor responses with lambrolizumab (anti-PD-1) in melanoma. N Engl J Med 2013;369:134.

[5] Hamanishi J, Mandai M, Ikeda T, Minami M, Kawaguchi A, Murayama T, et al. Safety and antitumor activity of anti-PD-1 antibody, nivolumab, in patients with platinum-resistant ovarian cancer. J Clin Oncol 2015;33:4015-22.

[6] Patnaik A, Kang SP, Rasco D, Papadopoulos KP, Elassaiss-Schaap J, Beeram M, et al. Phase I Study of pembrolizumab (MK-3475; Anti-PD-1 monoclonal antibody) in patients with advanced solid tumors. Clin Cancer Res 2015;21:4286-93.

[7] Rizvi NA, Mazières J, Planchard D, Stinchcombe TE, Dy GK, Antonia SJ, et al. Activity and safety of nivolumab, an anti-PD-1 immune checkpoint inhibitor for patients with advanced, refractory squamous non-small-cell lung cancer (CheckMate 063): a phase 2, single-arm trial. Lancet Oncol 2015;16:257-65.

[8] Garon EB, Rizvi NA, Hui R, Leighl N, Balmanoukian AS, Eder JP, et al. Pembrolizumab for the treatment of non-small-cell lung cancer. N Engl J Med 2015;372:2018-28.

[9] Brahmer J, Reckamp KL, Baas P, Crinò L, Eberhardt WEE, Poddubskaya E, et al Nivolumab versus Docetaxel in advanced squamous-cell non-small-cell lung cancer. N Engl J Med 2015;373:123-35.

[10] Gettinger SN, Horn L, Gandhi L, Spigel DR, Antonia SJ, Rizvi NA, et al. Overall survival and long-term safety of nivolumab (anti-programmed death 1 antibody, BMS-936558, ONO-4538) in patients with previously treated advanced non-small-cell lung cancer. J Clin Oncol 2015;33:2004-12.

[11] McDermott DF, Drake CG, Sznol M, Choueiri TK, Powderly JD, Smith DC, et al. Survival, durable response, and long-term safety in patients with previously treated advanced renal cell carcinoma receiving nivolumab. J Clin Oncol 2015;33:2013-20

[12] Robert C, Long GV, Brady B, Dutriaux C, Maio M, Mortier L, et al. Nivolumab in previously untreated melanoma without BRAF mutation. N Engl J Med 2015;372:320-30.

[13] Topalian SL, Sznol M, McDermott DF, Kluger HM, Carvajal RD, Sharfman WH, et al. Survival, durable tumor remission, and long-term safety in patients with advanced melanoma receiving nivolumab. J Clin Oncol 2014;32:1020.

[14] Ansell SM, Lesokhin AM, Borrello I, Halwani A, Scott EC, Gutierrez M, et al. PD1 Blockade with nivolumab in relapsed or refractory Hodgkin's lymphoma. N Engl J Med 2015;372:311-9.

[15] Motzer RJ, Rini BI, McDermott DF, Redman BG, Kuzel TM, Harrison MR, et al. Nivolumab for metastatic renal cell carcinoma: results of a randomized phase II trial. J Clin Oncol 2015;33:1430-7.

[16] Le DT, Uram JN, Wang H, Bartlett BR, Kemberling H, Eyring AD, et al. PD-1 Blockade in tumors with mismatch-repair deficiency. N Engl J Med 2015;372:2509-20.

[17] Postow MA, Chesney J, Pavlick AC, Robert C, Grossmann K, McDermott D, et al. Nivolumab and ipilimumab versus ipilimumab in untreated melanoma. N Engl J Med 2015;372:2006-17.

[18] Robert C, Schachter J, Long GV, Arance A, Grob JJ, Mortier L, et al. Pembrolizumab versus ipilimumab in advanced melanoma. N Engl J Med 2015;372:2521-32.

[19] Wolchok JD, Kluger H, Callahan MK, Postow MA, Rizvi NA, Lesokhin AM, et al. Nivolumab plus ipilimumab in advanced melanoma. N Engl J Med 2013;369:122.

[20] Borghaei H, Paz-Ares L, Horn L, Spigel DR, Steins M, Ready NE, et al. Nivolumab versus Docetaxel in advanced nonsquamous non-small-cell lung cancer. N Engl J Med 2015;373:1627-39.

[21] Okazaki T, Chikuma S, Iwai Y, Fagarasan S, Honjo T. A rheostat for immune responses: the unique properties of PD-1 and their advantages for clinical application. Nat Immunol 2013;14:1212-8.

[22] Keir ME, Butte MJ, Freeman GJ, Sharpe AH. PD-1 and its ligands in tolerance and immunity. Annu Rev Immunol 2008;26:677-704. 
[23] Granier C, Karaki S, Roussel H, Badoual C, Tran T, Anson M, et al. Cancer immunotherapy: rational and recent breakthroughs. Rev Med Interne 2016 [pii: S0248-8663(16)30413-1].

[24] Naidoo J, Page DB, Li BT, Connell LC, Schindler K, Lacouture ME, et al. Toxicities of the anti-PD-1 and anti-PD-L1 immune checkpoint antibodies. Ann Oncol 2015;26:2375.

[25] Champiat S, Lambotte O, Barreau E, Belkhir R, Berdelou A, Carbonnel F, et al. Management of immune checkpoint blockade dysimmune toxicities: a collaborative position paper. Ann Oncol Off J Eur Soc Med Oncol ESMO 2016;27:559-74.

[26] Bertrand A, Kostine M, Barnetche T, Truchetet ME, Schaeverbeke T. Immune related adverse events associated with anti-CTLA-4 antibodies: systematic review and meta-analysis. BMC Med 2015;13:211

[27] Polat P, Donofrio PD. Myasthenia gravis induced by nivolumab therapy in a patient with non-small-cell lung cancer: noteworthy cases. Muscle Nerve 2016;54:507.

[28] Lau KHV, Kumar A, Yang IH, Nowak RJ. Exacerbation of myasthenia gravis in a patient with melanoma treated with pembrolizumab: MG exacerbation after pembrolizumab. Muscle Nerve 2016;54:157-61.

[29] Loochtan AI, Nickolich MS, Hobson-Webb LD. Myasthenia gravis associated with ipilimumab and nivolumab in the treatment of small cell lung cancer. Muscle Nerve 2015;52:307-8.

[30] Jour G, Glitza IC, Ellis RM, Torres-Cabala CA, Tetzlaff MT, Li JY, et al. Autoimmune dermatologic toxicities from immune checkpoint blockade with anti-PD-1 antibody therapy: a report on bullous skin eruptions: bullous skin toxicities from anti-PD-1 antibody therapy. J Cutan Pathol 2016;43:688-96.

[31] Vandiver JW, Singer Z, Harshberger C. Severe hyponatremia and immune nephritis following an initial infusion of nivolumab. Target Oncol 2016;11:553-6.

[32] Hansen E, Sahasrabudhe D, Sievert L. A case report of insulin-dependent diabetes as immune-related toxicity of pembrolizumab: presentation, management and outcome. Cancer Immunol Immunother 2016;65:765-7.

[33] Alabed YZ, Aghayev A, Sakellis C, Van den Abbeele AD. Pancreatitis secondary to anti-programmed death receptor 1 immunotherapy diagnosed by FDG PET/CT. Clin Nucl Med 2015;40:e528-9.

[34] Danlos F-X, Pagès C, Baroudjian B, Vercellino L, Battistella M, Mimoun M, et al. Nivolumab-induced sarcoid-like granulomatous reaction in a patient with advanced melanoma. Chest 2016;149:e133-6.

[35] Koelzer VH, Rothschild SI, Zihler D, Wicki A, Willi B, Willi N, et al. Systemic inflammation in a melanoma patient treated with immune checkpoint inhibitors-an autopsy study. J Immunother Cancer 2016;4:13.

[36] Läubli H, Balmelli C, Bossard M, Pfister O, Glatz K, Zippelius A. Acute heart failure due to autoimmune myocarditis under pembrolizumab treatment for metastatic melanoma. J Immunother Cancer 2016;3(1) [Internet].

[37] Garel B, Kramkimel N, Trouvin A-P, Frantz C, Dupin N. Pembrolizumab-induced polymyalgia rheumatica in two patients with metastatic melanoma. Joint Bone Spine 2016, http://dx.doi.org/10.1016/j.jbspin.2016.01.007 [Epub ahead of print].

[38] Chan MM, Kefford RF, Carlino M, Clements A, Manolios N. Arthritis and tenosynovitis associated with the anti-PD1 antibody pembrolizumab in metastatic melanoma. J Immunother 2015;38:37-9.

[39] Salmon J-H, Lambrecht I, Brochot P, Grange F. A case of arthritis under pembrolizumab. Joint Bone Spine 2016, http://dx.doi. org/10.1016/j.jbspin.2016.03.003 [Epub ahead of print].

[40] Nair R, Gheith S, Nair SG. Immunotherapy-associated hemolytic anemia with pure red-cell aplasia. N Engl J Med 2016;374:1096-7.

[41] Kong BY, Micklethwaite KP, Swaminathan S, Kefford RF, Carlino MS. Autoimmune hemolytic anemia induced by anti-PD-1 therapy in metastatic melanoma. Melanoma Res 2016;26:202-4.

[42] Kanameishi S, Otsuka A, Nonomura Y, Fujisawa A, Endo Y, Kabashima K. Idiopathic thrombocytopenic purpura induced by nivolumab in a metastatic melanoma patient with elevated PD-1 expression on B cells. Ann Oncol 2016;27:546-7.

[43] Cappelli LC, Gutierrez AK, Baer AN, Albayda J, Manno RL, Haque U, et al. Inflammatory arthritis and sicca syndrome induced by nivolumab and ipilimumab. Ann Rheum Dis 2017;76:43-50.

[44] Ishikawa M, Oashi K. Case of hypophysitis caused by nivolumab. J Dermatol 2017:44:109-10.

[45] Nishino M, Giobbie-Hurder A, Hatabu H, Ramaiya NH, Hodi FS. Incidence of programmed cell death 1 inhibitor-related pneumonitis in patients with advanced cancer: a systematic review and meta-analysis. JAMA Oncol 2016;2: 1607-16.

[46] Carlos G, Anforth R, Chou S, Clements A, Fernandez-Peñas P. A case of bullous pemphigoid in a patient with metastatic melanoma treated with pembrolizumab. Melanoma Res 2015:25:265-8.

[47] Hwang SJE, Carlos G, Chou S, Wakade D, Carlino MS, Fernandez-Penas P. Bullous pemphigoid, an autoantibody-mediated disease, is a novel immune-related adverse event in patients treated with anti-programmed cell death 1 antibodies. Melanoma Res 2016;26:413-6.

[48] Beck KM, Dong J, Geskin LJ, Beltrani VP, Phelps RG, Carvajal RD, et al. Disease stabilization with pembrolizumab for metastatic acral melanoma in the setting of autoimmune bullous pemphigoid. J Immunother Cancer 2016;4:20.

[49] Corsello SM, Barnabei A, Marchetti P, De Vecchis L, Salvatori R, Torino F. Endocrine side effects induced by immune checkpoint inhibitors. J Clin Endocrinol Metab 2013;98:1361.
[50] Gaudy C, Clévy C, Monestier S, Dubois N, Préau Y, Mallet S, et al. Anti-PD1 pembrolizumab can induce exceptional fulminant type 1 diabetes. Diabetes Care 2015;38:e182-3.

[51] Hughes J, Vudattu N, Sznol M, Gettinger S, Kluger H, Lupsa B, et al. Precipitation of autoimmune diabetes with anti-PD-1 immunotherapy. Diabetes Care 2015;38:e55-7.

[52] Nishino M, Ramaiya NH, Awad MM, Sholl LM, Maattala JA, Taibi M, et al. PD-1 inhibitor-related pneumonitis in advanced cancer patients: radiographic patterns and clinical course. Clin Cancer Res 2016 2016, pii: clincanres.1320.

[53] Friedman CF, Proverbs-Singh TA, Postow MA. Treatment of the immunerelated adverse effects of immune checkpoint inhibitors: a review. JAMA Onco 2016.

[54] Belum VR, Benhuri B, Postow MA, Hellmann MD, Lesokhin AM, Segal NH, et al Characterisation and management of dermatologic adverse events to agents targeting the PD-1 receptor. Eur J Cancer 2016;60:12-25.

[55] Shoustari AN, Postow MA, Horvat TZ, Adel NG, Dang T, Chapman PB. Safety of pembrolizumab (pem) in patients (pts) who stopped ipilimumab (ipi) due to immune-related adverse events. ASCO Annual Meeting Proceedings. J Clin Oncol 2015;33(Suppl.) [abstr e20023].

[56] Wolchok JD, Hoos A, O’Day S, Weber JS, Hamid O, Lebbé C, et al. Guidelines for the evaluation of immune therapy activity in solid tumors: immune-related response criteria. Clin Cancer Res 2009;15:7412-20.

[57] Hodi FS, Hwu WJ, Kefford R, Weber JS, Daud A, Hamid O, et al. Evaluation of immune-related response criteria and recist v1.1 in patients with advanced melanoma treated with pembrolizumab. J Clin Oncol 2016;34:1510-7.

[58] Kyi C, Carvajal RD, Wolchok JD, Postow MA. Ipilimumab in patients with melanoma and autoimmune disease. J Immunother Cancer 2014;2:35.

[59] Bostwick AD, Salama AK, Hanks BA. Rapid complete response of metastatic melanoma in a patient undergoing ipilimumab immunotherapy in the setting of active ulcerative colitis. J Immunother Cancer 2015;3:19.

[60] Gettings EJ, Hackett CT, Scott TF. Severe relapse in a multiple sclerosis patient associated with ipilimumab treatment of melanoma. Mult Scler 2015;21:670.

[61] Johnson DB, Sullivan RJ, Ott PA, Carlino MS, Khushalani NI, Ye F, et al Ipilimumab therapy in patients with advanced melanoma and pre-existing autoimmune disorders. JAMA Oncol 2014;2:234-40.

[62] Maul LV, Weichenthal M, Kähler KC, Hauschild A. Successful anti-PD-1 antibody treatment in a metastatic melanoma patient with known severe autoimmune disease. J Immunother 2016;39:188-90.

[63] Bristol-Myers Squibb. Immune-mediated adverse reactions management guide; 2015 http://www.opdivoyervoyhcp.com/servlet/servlet. FileDownload?file=00Pi000000SRPWfEAP.

[64] Merck. A guide to monitoring patients during treatment with Keytruda; 2015 https://www.keytruda.com/static/pdf/adverse-reaction-management-tool.pdf.

[65] Boutros C, Tarhini A, Routier E, Lambotte O, Ladurie FL, Carbonnel F, et al. Safety profiles of anti-CTLA-4 and anti-PD-1 antibodies alone and in combination. Nat Rev Clin Oncol 2016;13:473-86.

[66] Eigentler TK, Hassel JC, Berking C, Aberle J, Bachmann O, Grünwald V, et al. Diagnosis, monitoring and management of immune-related adverse drug reactions of anti-PD-1 antibody therapy. Cancer Treat Rev 2016;45:7-18.

[67] Postow MA. Managing immune checkpoint-blocking antibody side effects ASCO Educational Book; 2015 [Accessed 21 Sept 2015] http://meetinglibrary. asco.org/content/115000076-156.

[68] Horvat TZ, Adel NG, Dang TO, Momtaz P, Postow MA, Callahan MK, et al. Immune-related adverse events, need for systemic immunosuppression, and effects on survival and time to treatment failure in patients with melanoma treated with Ipilimumab at memorial sloan kettering cancer center. J Clin Oncol 2015;33:3193.

[69] Weber JS, Yang JC, Atkins MB, Disis ML. Toxicities of immunotherapy for the practitioner. J Clin Oncol 2015;33:2092-9.

[70] NCCN: http://www.nccn.org/professionals/physician_gls/pdf/infections.pdf.

[71] Cooley L, Dendle C, Wolf J, Teh BW, Chen SC, Boutlis C, et al. Consensus guidelines for diagnosis, prophylaxis and management of Pneumocystis jirovecii pneumonia in patients with haematological and solid malignancies. Intern Med J 2014:1350-63.

[72] Pascolo S. Time to use a dose of chloroquine as an adjuvant to anti-cancer chemotherapies. Eur J Pharmacol 2016;771:139-44.

[73] Brunot A, et al. Anti-PD-1 tolerance after severe toxicity with ipilimumab therapy in metastatic melanoma patients. J Clin Oncol 2016;34(Suppl) [abstr 9551] http://meetinglibrary.asco.org/content/168789-176.

[74] Minn AJ, Wherry EJ. Combination cancer therapies with immune checkpoint blockade: convergence on interferon signaling. Cell 2016;165:272-5.

[75] Abu Eid R, Razavi GSE, Mkrtichyan M, Janik J, Khleif SN. Old-school chemotherapy in immunotherapeutic combination in cancer, a low-cost drug repurposed. Cancer Immunol Res 2016;4:377-82.

[76] Prieto PA, Reuben A, Cooper ZA, Wargo JA. Targeted therapies combined with immune checkpoint therapy. Cancer J Sudbury Mass 2016;22:138-46.

[77] Ribas A, Hodi FS, Callahan M, Konto C, Wolchok J. Hepatotoxicity with combination of vemurafenib and ipilimumab. N Engl J Med 2013;368:1365.

[78] Salama AKS, Postow MA, Salama JK. Irradiation and immunotherapy: from concept to the clinic. Cancer 2016;122:1659-71.

[79] Aspeslagh S, Postel-Vinay S, Rusakiewicz S, Soria J-C, Zitvogel L, Marabelle A. Rationale for anti-OX40 cancer immunotherapy. Eur J Cancer Oxf Engl 2016;1990:50-66.

[80] Serre R, Benzekry S, Padovani L, Meille C, André N, Ciccolini J, et al. Mathematica modeling of cancer immunotherapy and its synergy with radiotherapy. Cancer Res 2016;76:4931-40. 
[81] Kohrt HE, Tumeh PC, Benson D, Bhardwaj N, Brody J, Formenti S, et al. Cancer immunotherapy trials network (CITN). Immunodynamics: a cancer immunotherapy trials network review of immune monitoring in immunooncology clinical trials. J Immunother Cancer 2016;4:15.

[82] Taube JM, Klein A, Brahmer JR, Xu H, Pan X, Kim JH, et al. Association of PD-1, $\mathrm{PD}-1$ ligands, and other features of the tumor immune microenvironment with response to anti-PD-1 therapy. Clin Cancer Res 2014;20:5064-74.

[83] Breunis WB, Tarazona-Santos E, Chen R, Kiley M, Rosenberg SA, Chanock SJ. Influence of cytotoxic T lymphocyte-associated antigen 4 (CTLA4) common polymorphisms on outcome in treatment of melanoma patients with CTLA-4 blockade. J. Immunother 2008;31:586-90.

[84] Queirolo P, Morabito A, Laurent S, Lastraioli S, Piccioli P, Ascierto PA, et al. Association of CTLA-4 polymorphisms with improved overall survival in melanoma patients treated with CTLA-4 blockade: a pilot study. Cancer Invest 2013;31:336-45.

[85] Michot JM, Bigenwald C, Champiat S, Collins M, Carbonnel F, Postel-Vinay S, et al. Immune-related adverse events with immune checkpoint blockade: a comprehensive review. Eur J Cancer Oxf Engl 2016;1990:139-48.

[86] Spain L, Larkin J, Martin-Liberal J. Determining predictive factors for immune checkpoint inhibitor toxicity: response to letter to the editors "A case report of insulin-dependent diabetes as immune-related toxicity of pembrolizumab: presentation, management and outcome.". Cancer Immunol Immunother 2016;65:765-7.
[87] Dubin K, Callahan MK, Ren B, Khanin R, Viale A, Ling L, et al. Intestinal microbiome analyses identify melanoma patients at risk for checkpointblockade-induced colitis. Nat Commun 2016;7:10391.

[88] Schindler K, Harmankaya K, Kuk D. Correlation of absolute and relative eosinophil counts with immune-related adverse events in melanoma patients treated with ipilimumab. J Clin Oncol 2014;32(5s) [abstract 9096].

[89] Jaber SH, Cowen EW, Haworth LR, Booher SL, Berman DM, Rosenberg SA, et al. Skin reactions in a subset of patients with stage IV melanoma treated with anticytotoxic T-lymphocyte antigen 4 monoclonal antibody as a single agent. Arch Dermatol 2006;142:166.

[90] Callahan MK, Yang A. Tandon S, et al. Evaluation of serum IL-17 levels during ipilimumab therapy: correlation with colitis. J Clin Oncol 2011;29s:2505 [abstract 2505].

[91] Manson G, Norwood J, Marabelle A, Kohrt H, Houot R. Biomarkers associated with checkpoint inhibitors. Ann Oncol 2016;27:1199-206.

[92] Shahabi V, Berman D, Chasalow SD, Wang L, Tsuchihashi Z, Hu B, et al. Gene expression profiling of whole blood in ipilimumab-treated patients for identification of potential biomarkers of immune-related gastrointestinal adverse events. J Transl Med 2013;11:75

[93] Mariette X, Gottenberg J-E, Ravaud P, Combe B. Registries in rheumatoid arthritis and autoimmune diseases: data from the French registries. Rheumatol Oxf Engl 2011;50:222-9. 